\title{
FLAT CONNECTIONS AND RESONANCE VARIETIES: FROM RANK ONE TO HIGHER RANKS
}

\author{
DANIELA ANCA MĂCINIC ${ }^{1}$, ŞTEFAN PAPADIMA ${ }^{2}$, CLEMENT RADU POPESCU $^{3}$, \\ AND ALEXANDER I. SUCIU ${ }^{4}$
}

\begin{abstract}
Given a finitely-generated group $\pi$ and a linear algebraic group $G$, the representation variety $\operatorname{Hom}(\pi, G)$ has a natural filtration by the characteristic varieties associated to a rational representation of $G$. Its algebraic counterpart, the space of $\mathfrak{g}$-valued flat connections on a commutative, differential graded algebra $(A, d)$ admits a filtration by the resonance varieties associated to a representation of $\mathfrak{g}$. We establish here a number of results concerning the structure and qualitative properties of these embedded resonance varieties, with particular attention to the case when the rank 1 resonance variety decomposes as a finite union of linear subspaces. The general theory is illustrated in detail in the case when $\pi$ is either an Artin group, or the fundamental group of a smooth, quasi-projective variety.
\end{abstract}

\section{Contents}

1. Introduction 2

2. Flat connections and resonance varieties 6

3. Essentially rank 1 flat connections 11

4. Holonomy Lie algebras of differential graded algebras 15

5. Algebras with linear resonance 20

6. Artin groups 22

7. Quasi-projective manifolds 27

References $\quad 32$

2010 Mathematics Subject Classification. Primary 55N25, 55P62. Secondary 14F35, 20F36, 20 J05.

Key words and phrases. Resonance variety, characteristic variety, differential graded algebra, Lie algebra, flat connection, quasi-projective manifold, Artin group.

${ }^{1}$ Supported by a grant of the Romanian National Authority for Scientific Research, CNCS UEFISCDI, project number PN-II-RU-PD-2011-3-0149.

${ }^{2}$ Partially supported by the Romanian Ministry of National Education, CNCS-UEFISCDI, grant PNII-IDPCE-2012-4-0156.

${ }^{3}$ Supported by a grant of the Romanian National Authority for Scientific Research, CNCS-UEFISCDI, project number PN-II-RU-TE-2012-3-0492.

${ }^{4}$ Partially supported by NSF grant DMS-1010298 and NSA grant H98230-13-1-0225. 


\section{INTRODUCTION}

1.1. Representation varieties and flat connections. One way to understand a finitely generated group $\pi$ is to pick a complex linear algebraic group $G$, and look at the resulting representation variety, $\operatorname{Hom}(\pi, G)$. In the rank 1 case, one finds the character group $\mathbb{T}(\pi)=\operatorname{Hom}\left(\pi, \mathbb{C}^{\times}\right)$, which is simply the Pontryagin dual of the abelianization of $\pi$. In general, though, the $G$-representation variety is highly complicated, yet carries intricate information on the given group $\pi$, see for instance [5, 10, 13]. The Kapovich-Millson universality theorem from [13] describes the complexity of representation varieties in a precise way: the analytic germs away from the trivial representation $1 \in \operatorname{Hom}\left(\pi, \mathrm{PSL}_{2}\right)$, where $\pi$ runs through the family of Artin groups, are as complicated as arbitrary germs of affine varieties defined over $\mathbb{Q}$.

To make things simpler, it pays off to look at a closely related algebraic analogue of $\operatorname{Hom}(\pi, G)$. Given a commutative, differential graded algebra (for short, a cdga) $A=\left(A^{\bullet}, d\right)$ and a Lie algebra $\mathfrak{g}$, the tensor product $A \otimes \mathfrak{g}$ supports a graded, differential Lie algebra structure, with Lie bracket $[\alpha \otimes x, \beta \otimes y]=\alpha \beta \otimes[x, y]$ and differential $\partial(\alpha \otimes x)=d \alpha \otimes x$. A g $\mathfrak{g}$-valued flat connection on $A$ is a tensor $\omega \in A^{1} \otimes \mathfrak{g}$ that satisfies the Maurer-Cartan equation, $\partial \omega+\frac{1}{2}[\omega, \omega]=0$.

If both $A^{1}$ and $\mathfrak{g}$ are finite-dimensional, the set $\mathscr{F}(A, \mathfrak{g})$ of $\mathfrak{g}$-valued flat connections on $A$ is a closed subvariety of the affine space $A^{1} \otimes \mathfrak{g}$, containing the natural basepoint 0 . Moreover, if $A$ is a cdga model for a classifying space for $\pi$, and $\mathfrak{g}$ is the Lie algebra of $G$, then $\mathscr{F}(A, \mathfrak{g})$ may be viewed as an infinitesimal version of $\operatorname{Hom}(\pi, G)$. In the rank 1 case, things are again very simple: if $A$ is connected (i.e., $A^{0}=\mathbb{C} \cdot 1$ ), then $\mathscr{F}(A, \mathbb{C})=H^{1}(A)$. In higher ranks, we pay special attention to the Lie algebra $\mathfrak{s l}_{2}$ and its standard Borel subalgebra $\mathfrak{s o l}_{2}$, in which case the variety of flat connections is more amenable to concrete descriptions.

1.2. Cohomology jump loci. Both the representation variety and the variety of flat connections admit natural stratifications according to the dimensions of the relevant cohomology groups.

In the first case, let $X$ be a connected CW-complex with $\pi_{1}(X)=\pi$, and assume $X$ has finite $q$-type (i.e., $X$ has finite $q$-skeleton), for some $q \geqslant 1$. For each rational representation $\iota: G \rightarrow \mathrm{GL}(V)$, and each integer $i \leqslant q$, the sets

$$
\mathscr{V}_{r}^{i}(X, \iota)=\left\{\rho \in \operatorname{Hom}(\pi, G) \mid \operatorname{dim}_{\mathbb{C}} H^{i}\left(X, \iota_{\rho} V\right) \geqslant r\right\}
$$

are Zariski-closed in the representation variety. Together with the ambient space, these characteristic varieties (in degree $i$ and depth $r$ ) are homotopy-type invariants of $X$, encoding a plethora of information on the topology of the space $X$ and its covers, and reflecting certain geometric structures that $X$ may possess. These varieties (also known as the cohomology jumping loci, or the Green-Lazarsfeld sets in the algebro-geometric setting) have been the subject of much investigation, see for instance $[1,6,7,18,24]$.

In the second case, let $A$ be a connected cdga, and assume $A$ has finite $q$-type (i.e., $A^{\leqslant q}$ is finite-dimensional). Given a representation $\theta: \mathfrak{g} \rightarrow \mathfrak{g l}(V)$, one may turn the tensor product $A \otimes V$ into a cochain complex, with differential $d_{\omega}=d \otimes \operatorname{id}_{V}+\operatorname{ad}_{\omega}$ depending on $\omega$ and $\theta$, see (13). If both $\mathfrak{g}$ and $V$ are finite-dimensional, the sets

$$
\mathscr{R}_{r}^{i}(A, \theta)=\left\{\omega \in \mathscr{F}(A, \mathfrak{g}) \mid \operatorname{dim}_{\mathbb{C}} H^{i}\left(A \otimes V, d_{\omega}\right) \geqslant r\right\}
$$

are Zariski-closed in the parameter space for flat connections. These resonance varieties are more computable, and carry information in their own right about a space $X$ modeled by $A$.

The simplest situation is that of the rank 1 resonance varieties $\mathscr{R}_{r}^{i}(A)$, when $\mathfrak{g}=\mathbb{C}$ and $\theta=\operatorname{id}_{\mathbb{C}}$. In the particular case when $A=H^{\bullet}(X, \mathbb{C})$ is the cohomology algebra of a space $X$, 
endowed with the zero differential, these are the much-studied resonance varieties of $X$, denoted by $\mathscr{R}_{r}^{i}(X)$.

1.3. Overview of results. The resonance and characteristic varieties are intimately related, at least around the origin. More precisely, let $X$ be a space of finite $q$-type, and suppose Sullivan's de Rham model $\Omega^{\bullet}(X)$ has the same $q$-type as a cdga $A$ of finite $q$-type. Let $\iota: G \rightarrow \operatorname{GL}(V)$ be a rational representation, and let $\theta: \mathfrak{g} \rightarrow \mathfrak{g l}(V)$ be its tangent representation.

A foundational result from [6], extending work from [7], states the following: There is an analytic isomorphism between the germ of $\mathscr{F}(A, \mathfrak{g})$ at the trivial connection 0 and the germ of $\operatorname{Hom}(\pi, G)$ at the trivial representation 1 , which restricts to analytic isomorphisms $\mathscr{R}_{r}^{i}(A, \theta)_{(0)} \cong$ $\mathscr{V}_{r}^{i}(X, \iota)_{(1)}$, for all $i \leqslant q$ and $r \geqslant 0$.

In this note, we study the global structure of the resonance varieties sitting inside the variety of $\mathfrak{g}$-valued flat connections associated to a cdga $A$. When $A$ models $X$, and both objects satisfy appropriate finiteness assumptions, this approach provides valuable topological information on the space $X$. We obtain both general results, and explicit global descriptions for classes of cdga's related to important examples from group theory and algebraic geometry.

Our approach is motivated by the following natural question, which provides the main theme for the paper: Given a cdga $A$, how much information on its higher rank, embedded jump loci, $\left(\mathscr{F}(A, \mathfrak{g}), \mathscr{R}_{r}^{i}(A, \theta)\right)$, can be extracted from the knowledge of its rank 1 embedded jump loci, $\left(\mathscr{F}(A, \mathbb{C}), \mathscr{R}_{r}^{i}(A)\right)$ ?

1.4. A tangent cone formula. It is easy to see that the construction $\mathscr{F}(A, \mathfrak{g})$ is a bifunctor. We devote a large part of $\S 2$ to a general analysis of the functoriality properties of resonance varieties, which are much more delicate. This leads us to the following, rather general "tangent cone"-type result.

Theorem 1.1. Assume $A$ has the same q-type as $\Omega^{\bullet}(X)$, where $A$ and $X$ are of finite q-type. Then, for all $i \leqslant q$ and all $r \geqslant 0$, the tangent cone at 0 to the resonance variety $\mathscr{R}_{r}^{i}(A)$ is contained in $\mathscr{R}_{r}^{i}\left(H^{\bullet}(A)\right)$.

If, moreover, $A$ is defined over $\mathbb{Q}$, the identification $H^{1}\left(\Omega^{\bullet}(X)\right) \cong H^{1}(A)$ preserves $\mathbb{Q}$ structures, and $A$ has positive weights, then $\mathscr{R}_{r}^{i}(A) \subseteq \mathscr{R}_{r}^{i}\left(H^{\bullet}(A)\right)$, in the same range.

The first part of the theorem can be viewed as a rational homotopy analogue of a result of Libgober [14], which says the following: if $X$ is a connected, finite-type CW-complex, then, for all $i$ and $r$, the tangent cone at 1 to the characteristic variety $\mathscr{V}_{r}^{i}(X)$ is contained in $\mathscr{R}_{r}^{i}(X)$.

Examples of spaces $X$ of finite $q$-type equipped with a finite $q$-type cdga $A$ having the same $q$-type as $\Omega^{\bullet}(X)$ abound. For instance, if $X$ has finite $q$-type and is $q$-formal, we may take $A$ to be the cohomology algebra $H^{\bullet}(X, \mathbb{C})$, endowed with the zero differential. When $X$ is a compact Kähler manifold, and thus $X$ is formal, these properties hold even for $q=\infty$. Another important class of examples (where $q=\infty$ ) consists of irreducible, smooth, quasi-projective varieties (for short, quasi-projective manifolds), which come equipped with one of Morgan's Gysin models [16]. In both the $q$-formal and the quasi-projective cases, the additional conditions from Theorem 1.1 also hold. Finally, if $X=K(\pi, 1)$ is a classifying space for a finitely-generated nilpotent group $\pi$, we may take $A$ to be the cochain algebra of the Malcev-Lie algebra associated to $\pi$. We refer to [6] for more examples and details related to this topic.

1.5. Essentially rank 1 flat connections. Returning now to the general setup, assume $A$ is 1 finite and $\mathfrak{g}$ is finite-dimensional. Consider the closed subvariety through the origin, $\mathscr{F}^{1}(A, \mathfrak{g}) \subseteq$ 
$\mathscr{F}(A, \mathfrak{g})$, consisting of all tensors of the form $\eta \otimes g$ with $d \eta=0$. Given a finite-dimensional representation $\theta: \mathfrak{g} \rightarrow \mathfrak{g l}(V)$, we let $\Pi(A, \theta) \subseteq \mathscr{F}^{1}(A, \mathfrak{g})$ be the subvariety of all such tensors which also satisfy $\operatorname{det}(\theta(g))=0$. Note that $\mathscr{F}^{1}(A, \mathfrak{g})$ depends only on the vector spaces $H^{1}(A)$ and $\mathfrak{g}$, while $\Pi(A, \theta)$ depends only on $H^{1}(A)$ and the representation $\theta$. Both these varieties are easy to compute in any concrete situation. For instance, in the rank 1 case, $\mathscr{F}^{1}(A, \mathbb{C})=\mathscr{F}(A, \mathbb{C})$ and $\Pi(A, \theta)=\{0\}$. Using these notions, we show in $\S 3.2$ that the trace of higher rank depth 1 resonance on $\mathscr{F}^{1}(A, \mathfrak{g})$ is determined by rank 1 resonance, in the following precise way.

Theorem 1.2. Let $\omega=\eta \otimes g$ be an arbitrary element of $\mathscr{F}^{1}(A, \mathfrak{g})$. Then $\omega$ belongs to $\mathscr{R}_{1}^{k}(A, \theta)$ if and only if there is an eigenvalue $\lambda$ of $\theta(g)$ such that $\lambda \eta$ belongs to $\mathscr{R}_{1}^{k}(A)$. Moreover,

$$
\Pi(A, \theta) \subseteq \bigcap_{k: H^{k}(A) \neq 0} \mathscr{R}_{1}^{k}(A, \theta) .
$$

As a first application of this general result, we determine in $\S 4.7$ the germs at 1 of embedded depth 1 characteristic varieties, for a finitely-generated nilpotent group $\pi$, in the case when $G$ is either semisimple of rank one, or one of its Borel subgroups. Given a rational representation $\iota: G \rightarrow \operatorname{GL}(V)$, with tangent map $\theta$, we show that $\mathscr{F}(A, \mathfrak{g})=\mathscr{F}^{1}(A, \mathfrak{g})$, where $A=\mathcal{C} \cdot(\mathfrak{n})$ is the Chevalley-Eilenberg cochain algebra of the Malcev-Lie algebra $\mathfrak{n}$ associated to $\pi$. Moreover,

$$
\mathscr{R}_{1}^{k}(\mathcal{C} \cdot(\mathfrak{n}), \theta)=\Pi(\mathcal{C} \cdot(\mathfrak{n}), \theta)
$$

if $k \leqslant \operatorname{dim} \mathfrak{n}$ and is empty otherwise.

1.6. Holonomy Lie algebras. In $\S 4$, we consider the Lie analogue of resonance varieties, expanding on the approach from [7]. Given a Lie algebra $\mathfrak{h}$, and a representation $\theta: \mathfrak{g} \rightarrow \mathfrak{g l}(V)$ of another Lie algebra, the corresponding resonance varieties are defined as

$$
\mathscr{R}_{r}^{i}(\mathfrak{h}, \theta)=\left\{\rho \in \operatorname{Hom}_{\text {Lie }}(\mathfrak{h}, \mathfrak{g}) \mid \operatorname{dim}_{\mathbb{C}} H^{i}\left(\mathfrak{h}, V_{\theta \circ \rho}\right) \geqslant r\right\} .
$$

We relate this notion of resonance to the previous one, under the assumption that both $\mathfrak{g}$ and $V$ have finite dimension. Given a connected cdga $A$ with $A^{1}$ finite-dimensional, we define in a functorial way the holonomy Lie algebra $\mathfrak{h}(A)$, as a certain quotient of the free Lie algebra on the dual vector space $A_{1}=\left(A^{1}\right)^{*}$. We then prove the following result.

Theorem 1.3. The natural linear isomorphism $\psi: A^{1} \otimes \mathfrak{g} \cong \operatorname{Hom}\left(A_{1}, \mathfrak{g}\right)$ restricts to an isomorphism $\psi: \mathscr{F}(A, \mathfrak{g}) \cong \operatorname{Hom}_{\text {Lie }}(\mathfrak{h}(A), \mathfrak{g})$, and induces isomorphisms $\mathscr{R}_{r}^{i}(A, \theta) \cong \mathscr{R}_{r}^{i}(\mathfrak{h}(A), \theta)$, for $i \leqslant 1$ and $r \geqslant 0$.

Furthermore, if $A=\mathcal{C} \cdot(\mathfrak{f})$, where $\mathfrak{f}$ is a Lie algebra of finite dimension, then $\mathfrak{h}(A)=\mathfrak{f}$, and $\psi$ induces isomorphisms $\mathscr{R}_{r}^{i}(A, \theta) \cong \mathscr{R}_{r}^{i}(\mathfrak{f}, \theta)$, for all $i, r \geqslant 0$.

We also show that the non-triviality of the depth 1 , degree 1 resonance varieties is detected in the rank 1 case by the Lie algebra $\mathfrak{s o l}_{2}$, and by the 3 -dimensional Heisenberg Lie algebra $\mathfrak{h e i s}_{3}$. More precisely, $\mathscr{R}_{1}^{1}(A) \nsubseteq\{0\}$ if and only if $\operatorname{Hom}_{\text {Lie }}\left(\mathfrak{h}(A), \mathfrak{s o l}_{2}\right)$ contains a surjection, while $\mathscr{R}_{1}^{1}\left(H^{\bullet}(A)\right) \nsubseteq\{0\}$ if and only if $\operatorname{Hom}_{\text {Lie }}\left(\mathfrak{h}(A), \mathfrak{h e i s}_{3}\right)$ contains a surjection.

1.7. Linear resonance. Starting in $\S 5$, and for the rest of the paper, we focus our attention on the class of cdga's $A$ for which the rank 1 resonance variety $\mathscr{R}_{1}^{1}(A)$ decomposes as a finite union of linear subspaces. From this point on, we assume the bare minimum in terms of finiteness conditions that are needed for our approach to work well: $A$ will be a connected, 1-finite cdga, and $\theta$ will be a finite-dimensional representation of a Lie algebra $\mathfrak{g}$ of finite dimension. 
It turns out that, under some additional hypotheses on $A$ and $\mathfrak{g}$, the structure of both the parameter space $\mathscr{F}(A, \mathfrak{g})$ and of the higher-rank resonance varieties $\mathscr{R}_{1}^{1}(A, \theta)$ is determined by the decomposition of the rank 1 resonance variety into linear irreducible components. More precisely, we prove the following structural result.

Theorem 1.4. Suppose $\mathscr{R}_{1}^{1}(A)=\bigcup_{C \in \mathscr{C}} C$, a finite union of linear subspaces. For each $C \in \mathscr{C}$, let $A_{C}$ denote the sub-cdga of the truncation $A^{\leqslant 2}$ defined by $A_{C}^{1}=C$ and $A_{C}^{2}=A^{2}$. Then, for any Lie algebra $\mathfrak{g}$,

$$
\mathscr{F}(A, \mathfrak{g}) \supseteq \mathscr{F}^{1}(A, \mathfrak{g}) \cup \bigcup_{0 \neq C \in \mathscr{C}} \mathscr{F}\left(A_{C}, \mathfrak{g}\right),
$$

where each $\mathscr{F}\left(A_{C}, \mathfrak{g}\right)$ is Zariski-closed in $\mathscr{F}(A, \mathfrak{g})$. Moreover, if $A$ has zero differential, $A^{1}$ is non-zero, and $\mathfrak{g}=\mathfrak{s l}_{2}$ or $\mathfrak{s o l}_{2}$, then (5) holds as an equality, and, for any $\theta$,

$$
\mathscr{R}_{1}^{1}(A, \theta)=\Pi(A, \theta) \cup \bigcup_{0 \neq C \in \mathscr{C}} \mathscr{F}\left(A_{C}, \mathfrak{g}\right) .
$$

For instance, consider the case when $X$ is a 1-finite, 1-formal space, and $A=H^{\bullet}(X, \mathbb{C})$ with differential $d=0$. Then, as shown in [7], the resonance variety $\mathscr{R}_{1}^{1}(A)$ is linear. By a result from [6], Gysin models of quasi-projective manifolds also have the linearity property for $\mathscr{R}_{1}^{1}(A)$, but the differential in this case may well be non-zero.

1.8. Artin groups. In $\S 6$, we analyze the Kapovich-Millson family of representation varieties, near the origin. Given a finite simplicial graph $\Gamma$, with edges labeled by integers greater than 1, there is an associated Artin group $\pi_{\Gamma}$; if all labels are equal to 2, the group $\pi_{\Gamma}$ is called a right-angled Artin group. According to [13], every Artin group $\pi_{\Gamma}$ has a classifying space $X_{\Gamma}$ which is 1-finite and 1-formal. For the purpose of computing the embedded degree 1 resonance varieties, we may model this space by the cdga $A_{\Gamma}=\left(H^{\bullet}\left(\pi_{\Gamma}, \mathbb{C}\right), d=0\right)$, to which Theorem 1.4 applies.

We use holonomy Lie algebras, as well as results from [17] and [7] to describe explicitly the global irreducible decompositions for $\mathscr{F}\left(A_{\Gamma}, \mathfrak{g}\right)$ and $\mathscr{R}_{1}^{1}\left(A_{\Gamma}, \theta\right)$, in terms of the "odd contraction" of the labeled graph $\Gamma$ and a representation $\theta$ of $\mathfrak{g}=\mathfrak{s l}_{2}$. For instance, if $\pi_{\Gamma}$ is a right-angled Artin group, then

$$
\mathscr{F}\left(A_{\Gamma}, \mathfrak{s l}_{2}\right)=\bigcup_{\mathrm{W} \subseteq \mathrm{V}} S_{\mathrm{W}}
$$

where $W$ runs through the subsets of the vertex set of $\Gamma$, maximal with respect to an order $\leqslant$ defined in terms of the connected components of the induced subgraph $\Gamma_{\mathrm{W}}$, while $S_{\mathrm{W}}$ is a certain combinatorially defined, closed subvariety of $\mathbb{C}^{\mathrm{W}} \otimes \mathfrak{s l}_{2}$, in fact, a product of cones over varieties of the form $\mathbb{P}^{n} \times \mathbb{P}^{2}$, see (39).

Taking germs at 0 , these results give a concrete way to compute the germs at the origin for both $\operatorname{Hom}\left(\pi_{\Gamma}, \mathrm{PSL}_{2}\right)$ and $\mathscr{V}_{1}^{1}\left(X_{\Gamma}, \iota\right)$, where $\iota$ is an arbitrary rational representation of $\mathrm{PSL}_{2}$. Our explicit description of the singularity germ $\operatorname{Hom}\left(\pi_{\Gamma}, \mathrm{PSL}_{2}\right)_{(1)}$ complements the qualitative quadraticity restriction on this germ given by Kapovich and Millson in [13].

For an arbitrary Lie algebra $\mathfrak{g}$, we show that the variety $\mathscr{F}\left(A_{\Gamma}, \mathfrak{g}\right)$ contains the union of the subvarieties $S_{\mathrm{W}}$, defined in a similar manner as above. If $\mathfrak{g}$ is semisimple and different from $\mathfrak{s l}_{2}$, though, we show by example that this containment can be strict. 
1.9. Quasi-projective manifolds. A basic object of study in algebraic geometry is the class of quasi-projective varieties. In $\S 7$, we apply our machinery to quasi-projective manifolds.

Let $X$ be such a manifold, and assume $b_{1}(X)>0$. In this case, the irreducible decomposition of $\mathscr{R}_{1}^{1}(A)$, for a suitable Gysin model $A$ of $X$, can be described in geometric terms. The irreducible components are all linear, and they are indexed by a finite list, denoted $\mathscr{E}_{X}$, of equivalence classes of regular "admissible" maps $f: X \rightarrow S$, where the quasi-projective manifolds $S$ are 1-dimensional, and have negative Euler characteristic. More precisely, it follows from [6] that

$$
\mathscr{R}_{1}^{1}(A)=\{0\} \cup \bigcup_{f \in \mathscr{E}_{X}} f^{*}\left(H^{1}(A(S))\right),
$$

where $A(S)$ is Morgan's Gysin model of $S$. If $X$ is also 1-formal, it follows from [7] that the same decomposition formula holds with Gysin models replaced by cohomology rings, endowed with the zero differential.

In the 1-formal situation, we use again Theorem 1.4 to find explicit (global) irreducible decompositions for $\mathscr{F}\left(H^{\bullet}(X, \mathbb{C}), \mathfrak{g}\right)$ and $\mathscr{R}_{1}^{1}\left(H^{\bullet}(X, \mathbb{C}), \theta\right)$ in the case when $\mathfrak{g}=\mathfrak{s l}_{2}$, solely in terms of the set $\mathscr{E}_{X}$ and of the representation $\theta$. In the (much more delicate) general case, we are able to show that $\mathscr{F}(A, \mathfrak{g})=\mathscr{F}^{1}(A, \mathfrak{g})$ and $\mathscr{R}_{1}^{1}(A, \theta)=\Pi(A, \theta)$, for an arbitrary Gysin model $A$ of $X$, and for a representation $\theta$ of $\mathfrak{g}=\mathfrak{s l}_{2}$ or $\mathfrak{s o l}_{2}$, provided that $\mathscr{R}_{1}^{1}(X)=\{0\}$.

In [22], we apply this machinery to the case when $X$ is the complement of a complex hyperplane arrangement. Exploiting the known decomposition into irreducible components of $\mathscr{R}_{1}^{1}(X)$, and the fact that $X$ is formal, we determine the irreducible decomposition of $\mathscr{F}\left(H^{\bullet}(X, \mathbb{C}), \mathfrak{s l}_{2}\right)$, and then relate this decomposition to the algebraic monodromy of the Milnor fibration of the arrangement.

1.10. Notations and conventions. Throughout, we work over $\mathbb{C}$. Affine varieties and analytic germs will always be reduced. For a point $v$ on an affine variety $\mathscr{V}$, we let $\mathscr{V}_{(v)}$ denote the associated germ.

We denote vector space duals by $V^{*}$. For a graded vector space $A^{\bullet}$, we write $A \cdot{ }^{*}=\left(A^{\bullet}\right)^{*}$. Given two vector spaces, $V$ and $W$, we denote by $\operatorname{Hom}^{1}(V, W)$ the subset of those linear maps from $V$ to $W$ whose image is at most 1-dimensional. We also let $\psi: V \otimes W \rightarrow \operatorname{Hom}\left(V^{*}, W\right)$ be the linear map given by $\psi(v \otimes w)\left(u^{*}\right)=\left\langle v, u^{*}\right\rangle w$, where $\langle\cdot, \cdot\rangle$ stands for the duality pairing. (This map is always injective, and it is an isomorphism when $\operatorname{dim} V<\infty$.)

\section{Flat CONNECTIONS AND RESONANCE VARIETIES}

We start by reviewing the two basic algebraic constructions in the title of this section, paying special attention to their functoriality properties, and to the rank 1 case.

2.1. Representation varieties and jump loci. Let $X$ be a path-connected space, and let $\pi=\pi_{1}(X)$ be its fundamental group. We say that $X$ is of finite $q$-type (or q-finite), for some integer $q \geqslant 1$, if $X$ has the homotopy type of a CW-complex with finite $q$-skeleton.

Now let $G$ be a linear algebraic group. The set of group homomorphisms from $\pi$ to $G$, denoted $\operatorname{Hom}(\pi, G)$, is called the $G$-representation variety of $\pi$. This set comes with an obvious basepoint: the trivial representation, $1 \in \operatorname{Hom}(\pi, G)$. If the space $X$ is 1-finite, or, equivalently, the group $\pi$ is finitely generated, then $\operatorname{Hom}(\pi, G)$ has a natural affine structure.

Next, fix a rational representation $\iota: G \rightarrow \mathrm{GL}(V)$. By definition, the characteristic varieties (or, cohomology jump loci) of $X$, in degree $i \geqslant 0$ and depth $r \geqslant 0$, and with respect to the 
representation $\iota$, are the sets

$$
\mathscr{V}_{r}^{i}(X, \iota)=\left\{\rho \in \operatorname{Hom}(\pi, G) \mid \operatorname{dim}_{\mathbb{C}} H^{i}\left(X, \iota_{\rho} V\right) \geqslant r\right\},
$$

where $\iota_{\iota \rho} V$ denotes the left $\pi$-module defined by the representation $\iota \circ \rho: \pi \rightarrow \operatorname{GL}(V)$. If the space $X$ is $q$-finite, the jump loci $\mathscr{V}_{r}^{i}(X, \iota)$ are Zariski-closed subsets of the representation variety $\operatorname{Hom}(\pi, G)$, for all $i \leqslant q$ and $r \geqslant 0$.

The most basic (and best understood) case is the rank 1 case, which corresponds to the choices $G=\mathbb{C}^{\times}, V=\mathbb{C}$, and $\iota: \mathbb{C}^{\times} \stackrel{\equiv}{\rightrightarrows} \mathrm{GL}_{1}(\mathbb{C})$. The respective jump loci, denoted simply by $\mathscr{V}_{r}^{i}(X)$, are subsets of the character group $\mathbb{T}(\pi):=\operatorname{Hom}\left(\pi, \mathbb{C}^{\times}\right)$.

Remark 2.1. Let $\chi: \pi \rightarrow \mathrm{GL}(V)$ be a finite-dimensional representation of the group $\pi=\pi_{1}(X)$, defining a left $\pi$-module ${ }_{\chi} V$, and let $V_{\chi}^{*}$ be the dual (right) $\pi$-module. We then have a natural duality isomorphism, $H^{\bullet}\left(X,{ }_{\chi} V\right) \stackrel{\simeq}{\longrightarrow} H \cdot\left(X, V_{\chi}^{*}\right)^{*}$, see for instance Lemma 8.5 from preprint v.1 of [6]. Hence, when defining the rank 1 characteristic varieties $\mathscr{V}_{r}^{i}(X)$, cohomology may be replaced by homology, as done, for instance, in $[18,20]$.

2.2. Flat connections. We switch now to a more algebraic (yet very much related) framework. Let $A=\left(A^{\bullet}, d\right)$ be a commutative, differential graded algebra (for short, a cdga) over $\mathbb{C}$. We say that $A$ is of finite $q$-type (or $q$-finite), for some $1 \leqslant q \leqslant \infty$, if $A$ is connected (that is, $A^{0}$ is the $\mathbb{C}$-span of the unit 1 ), and $A^{\leqslant q}$ is finite-dimensional.

Next, let $\mathfrak{g}$ be a Lie algebra over $\mathbb{C}$. Given any cdga $A$, consider the tensor product $A \otimes \mathfrak{g}$. On this vector space, we may define a Lie bracket given by $[\alpha \otimes x, \beta \otimes y]=\alpha \beta \otimes[x, y]$, and a differential given by $\partial(\alpha \otimes x)=d \alpha \otimes x$. This construction produces a graded, differential Lie algebra (for short, dgla), denoted $A \otimes \mathfrak{g}=\left(A^{\bullet} \otimes \mathfrak{g}, \partial\right)$. Moreover, the construction is functorial in both $A$ and $\mathfrak{g}$.

The algebraic analogue of the $G$-representation variety $\operatorname{Hom}(\pi, G)$ is the set of $\mathfrak{g}$-valued flat connections on $A$. By definition, this is the set $\mathscr{F}(A, \mathfrak{g})$ of degree 1 elements in $A \otimes \mathfrak{g}$ that satisfy the Maurer-Cartan equation,

$$
\partial \omega+\frac{1}{2}[\omega, \omega]=0 .
$$

A typical element in $A^{1} \otimes \mathfrak{g}$ is of the form $\omega=\sum_{i} \eta_{i} \otimes g_{i}$, with $\eta_{i} \in A^{1}$ and $g_{i} \in \mathfrak{g}$; the flatness condition amounts to

$$
\sum_{i} d \eta_{i} \otimes g_{i}+\sum_{i<j} \eta_{i} \eta_{j} \otimes\left[g_{i}, g_{j}\right]=0 .
$$

The set of flat connections depends functorially on both $A$ and $\mathfrak{g}$, and comes with an obvious basepoint: the trivial connection, $0 \in \mathscr{F}(A, \mathfrak{g})$. Now suppose that both $A^{1}$ and $\mathfrak{g}$ are finitedimensional. It is then readily seen that $\mathscr{F}(A, \mathfrak{g})$ is a Zariski-closed subset of the affine space $A^{1} \otimes \mathfrak{g}$, and thus carries a natural affine structure.

The simplest situation is the rank one case, for which $\mathfrak{g}=\mathbb{C}$. In this case, the space $\mathscr{F}(A, \mathbb{C})$ may be identified with the vector space $Z^{1}(A)=\left\{\alpha \in A^{1} \mid d \alpha=0\right\}$. In particular, if $d=0$, then $\mathscr{F}(A, \mathbb{C})=A^{1}$.

Remark 2.2. The terminology we use here comes from differential geometry. Indeed, if $\Omega^{\bullet}(X)$ is the algebra of differential forms on a connected, smooth manifold $X$, and $\mathfrak{g}$ is the Lie algebra of a Lie group $G$, then $\mathscr{F}\left(\Omega^{\bullet}(X), \mathfrak{g}\right)$ may be identified with the space of flat connections on the principal (trivial) bundle $X \times G \rightarrow X$. When $G$ is a linear algebraic group and $\pi_{1}(X)$ is finitely 
generated, the functorial monodromy, $\mathscr{F}\left(\Omega^{\bullet}(X), \mathfrak{g}\right) \rightarrow \operatorname{Hom}\left(\pi_{1}(X), G\right)$, determines the germ at 1 of the representation variety. For a detailed treatment of this topic, we refer to [10].

2.3. Aomoto complex. Returning now to the general situation, let $V$ be a complex vector space, and let $\theta: \mathfrak{g} \rightarrow \mathfrak{g l}(V)$ be a morphism of Lie algebras, also known as a representation of the Lie algebra $\mathfrak{g}$ in $V$, or a $\mathfrak{g}$-module structure on $V$. For each flat connection $\omega \in \mathscr{F}(A, \mathfrak{g})$, we make the tensor product $A \otimes V$ into a cochain complex,

$$
\left(A \otimes V, d_{\omega}\right): A^{0} \otimes V \stackrel{d_{\omega}}{\longrightarrow} A^{1} \otimes V \stackrel{d_{\omega}}{\longrightarrow} A^{2} \otimes V \stackrel{d_{\omega}}{\longrightarrow} \cdots,
$$

using as differential the covariant derivative $d_{\omega}=d \otimes \operatorname{id}_{V}+\operatorname{ad}_{\omega}$. Here, the adjoint operator ad ${ }_{\omega}$ is defined by the semi-direct product Lie algebra $V \rtimes_{\theta} \mathfrak{g}$. Explicitly, if $\omega=\sum_{i} \eta_{i} \otimes g_{i}$, then

$$
d_{\omega}(\alpha \otimes v)=d \alpha \otimes v+\sum_{i} \eta_{i} \alpha \otimes \theta\left(g_{i}\right) v,
$$

for all $\alpha \in A^{i}$ and $v \in V$. It is readily checked that the flatness condition on $\omega$ insures that $d_{\omega}^{2}=0$.

The cochain complex (12), which we will call the Aomoto complex, enjoys the following naturality property. Given a morphism $\varphi: A \rightarrow A^{\prime}$ of cdga's, set $\omega^{\prime}=\varphi \otimes \operatorname{id}_{\mathfrak{g}}(\omega)$; then $\varphi \otimes \operatorname{id}_{V}:\left(A \otimes V, d_{\omega}\right) \rightarrow\left(A^{\prime} \otimes V, d_{\omega^{\prime}}\right)$ is a cochain map.

Note that $A^{\bullet} \otimes V$ is a free graded left $A^{\bullet}$-module. Moreover, it is easily checked that the Aomoto complex is a dg-module over the cdga $\left(A^{\bullet}, d\right)$. In particular, $H^{\bullet}\left(A^{\bullet} \otimes V, d_{\omega}\right)$ acquires a natural graded $H^{\bullet}(A)$-module structure.

The next lemma provides a simple interpretation of the 0-th cohomology of the Aomoto complex.

Lemma 2.3. Let $A$ a be a connected cdga, and let $\theta: \mathfrak{g} \rightarrow \mathfrak{g l}(V)$ be a representation. Pick a basis $\left\{\eta_{i}\right\}$ for $A^{1}$, and suppose $\omega=\sum_{i} \eta_{i} \otimes g_{i} \in \mathscr{F}(A, \mathfrak{g})$ is a flat connection. Then

$$
H^{0}\left(A \otimes V, d_{\omega}\right)=\bigcap_{i} \operatorname{ker}\left(\theta\left(g_{i}\right)\right) .
$$

Proof. From the definitions, we have that $H^{0}\left(A \otimes V, d_{\omega}\right)=\operatorname{ker}\left(d_{\omega}: A^{0} \otimes V \rightarrow A^{1} \otimes V\right)$, where $d_{\omega}(1 \otimes v)=\sum_{i} \eta_{i} \otimes \theta\left(g_{i}\right) v$. The conclusion follows at once.

2.4. Resonance varieties. More generally, we may compute the cohomology of the Aomoto complex in any fixed degree, and record the set of flat connections for which the dimension of this vector space jumps above a prescribed value. This leads to defining the resonance varieties of a cdga $A$, with coefficients given by a representation $\theta: \mathfrak{g} \rightarrow \mathfrak{g l}(V)$ as

$$
\mathscr{R}_{r}^{i}(A, \theta)=\left\{\omega \in \mathscr{F}(A, \mathfrak{g}) \mid \operatorname{dim}_{\mathbb{C}} H^{i}\left(A \otimes V, d_{\omega}\right) \geqslant r\right\} .
$$

If $A$ is $q$-finite, and both $\mathfrak{g}$ and $V$ are finite-dimensional, the sets $\mathscr{R}_{r}^{i}(A, \theta)$ are Zariski-closed subsets of $\mathscr{F}(A, \mathfrak{g})$, for all $i \leqslant q$ and $r \geqslant 0$.

In the rank one case, we will simply write $\mathscr{R}_{r}^{i}(A):=\mathscr{R}_{r}^{i}\left(A, \mathrm{id}_{\mathbb{C}}\right)$ for the corresponding resonance varieties, viewed as algebraic subsets of $H^{1}(A)=Z^{1}(A)$. If $d=0$, the varieties $\mathscr{R}_{r}^{i}(A)$ are homogeneous subsets of $A^{1}$. In general, though, the varieties $\mathscr{R}_{r}^{i}(A)$ are not homogeneous.

The resonance varieties of a path-connected space $X$ are traditionally defined as the rank1 resonance varieties of its cohomology algebra (endowed with the zero differential); that is, $\mathscr{R}_{r}^{i}(X):=\mathscr{R}_{r}^{i}\left(H^{\bullet}(X, \mathbb{C})\right)$. More generally, we set $\mathscr{R}_{r}^{i}(X, \theta):=\mathscr{R}_{r}^{i}\left(H^{\bullet}(X, \mathbb{C}), \theta\right)$, for a representation $\theta: \mathfrak{g} \rightarrow \mathfrak{g l}(V)$. 
To avoid trivialities, we will assume from now on that $V \neq 0$, in which case

$$
0 \in \mathscr{R}_{1}^{i}(A, \theta) \Leftrightarrow 0 \in \mathscr{R}_{1}^{i}(A) \Leftrightarrow H^{i}(A) \neq 0 .
$$

Here is a simple situation where the resonance variety $\mathscr{R}_{1}^{1}(A, \theta)$ can be identified explicitly.

Proposition 2.4. Let $A \cdot$ be a 2 -finite cdga, and let $\theta: \mathfrak{g} \rightarrow \mathfrak{g l}(V)$ be a finite-dimensional representation. If $A^{>2}=0$ and $\chi\left(H^{\bullet}(A)\right)<0$, then $\mathscr{R}_{1}^{1}(A, \theta)=\mathscr{F}(A, \mathfrak{g})$.

Proof. Let $\omega \in \mathscr{F}(A, \mathfrak{g})$ be an arbitrary element, and suppose $H^{1}\left(A \otimes V, d_{\omega}\right)=0$. Then clearly $\chi\left(H^{\bullet}\left(A \otimes V, d_{\omega}\right)\right) \geqslant 0$. On the other hand,

$$
\chi\left(H^{\bullet}\left(A \otimes V, d_{\omega}\right)\right)=\chi\left(A^{\bullet}\right) \cdot \operatorname{dim} V=\chi\left(H^{\bullet}(A)\right) \cdot \operatorname{dim} V<0,
$$

a contradiction. Thus, $\omega \in \mathscr{R}_{1}^{1}(A, \theta)$, and we are done.

2.5. Functoriality of resonance. Let us now discuss the functoriality properties of the resonance varieties $\mathscr{R}_{r}^{i}(A, \theta)$ attached to a cdga $A$ and a representation $\theta: \mathfrak{g} \rightarrow \mathfrak{g l}(V)$.

First, let $f: \mathfrak{g} \rightarrow \mathfrak{g}^{\prime}$ be a morphism of Lie algebras. Clearly, the map $\bar{f}=\operatorname{id}_{A^{1}} \otimes f: A^{1} \otimes \mathfrak{g} \rightarrow$ $A^{1} \otimes \mathfrak{g}^{\prime}$ restricts to a map $\bar{f}: \mathscr{F}(A, \mathfrak{g}) \rightarrow \mathscr{F}\left(A, \mathfrak{g}^{\prime}\right)$. Hence, if $\theta^{\prime}: \mathfrak{g}^{\prime} \rightarrow \mathfrak{g l}(V)$ is a representation such that $\theta=\theta^{\prime} \circ f, \omega \in A^{1} \otimes \mathfrak{g}$, and $\omega^{\prime}=\bar{f}(\omega)$, then $d_{\omega}=d_{\omega^{\prime}}$ and

$$
\mathscr{R}_{r}^{i}(A, \theta)=\bar{f}^{-1}\left(\mathscr{R}_{r}^{i}\left(A, \theta^{\prime}\right)\right),
$$

for all $i \geqslant 0$ and $r \geqslant 0$.

Remark 2.5. By taking $f=\theta$ in formula (16), we see that, from the point of view of the resonance varieties of $A$, the case when $\theta^{\prime}$ is the identity of $\mathfrak{g l}(V)$ determines all others; that is to say, $\mathscr{R}_{r}^{i}(A, \theta)=\bar{\theta}^{-1}\left(\mathscr{R}_{r}^{i}\left(A, \operatorname{id}_{\mathfrak{g r}(V)}\right)\right)$.

Next, let $\varphi: A \rightarrow A^{\prime}$ be a morphism of cdga's. The map $\varphi \otimes \operatorname{id}_{\mathfrak{g}}: A^{1} \otimes \mathfrak{g} \rightarrow A^{1} \otimes \mathfrak{g}$ restricts to a map $\bar{\varphi}: \mathscr{F}(A, \mathfrak{g}) \rightarrow \mathscr{F}\left(A^{\prime}, \mathfrak{g}\right)$. The next lemma shows that the resonance varieties of $A$ and $A^{\prime}$ agree, roughly in the range for which $\varphi$ is an isomorphism.

Lemma 2.6. Suppose $\varphi: A^{\bullet} \rightarrow A^{\prime}$ is an isomorphism up to degree $q$, and a monomorphism in degree $q+1$, for some $q \geqslant 0$.

(1) If $q \geqslant 1$, the map $\bar{\varphi}$ is an isomorphism which identifies $\mathscr{R}_{r}^{i}(A, \theta)$ with $\mathscr{R}_{r}^{i}\left(A^{\prime}, \theta\right)$ for each $i \leqslant q$, and sends $\mathscr{R}_{r}^{q+1}(A, \theta)$ into $\mathscr{R}_{r}^{q+1}\left(A^{\prime}, \theta\right)$, for all $r \geqslant 0$.

(2) If $q=0$, the map $\bar{\varphi}$ is an embedding which identifies $\mathscr{R}_{r}^{0}(A, \theta)$ with $\mathscr{R}_{r}^{0}\left(A^{\prime}, \theta\right) \cap \mathscr{F}(A, \mathfrak{g})$, and sends $\mathscr{R}_{r}^{1}(A, \theta)$ into $\mathscr{R}_{r}^{1}\left(A^{\prime}, \theta\right)$, for all $r \geqslant 0$.

Proof. The fact that the map $\bar{\varphi}: \mathscr{F}(A, \mathfrak{g}) \rightarrow \mathscr{F}\left(A^{\prime}, \mathfrak{g}\right)$ is an isomorphism, respectively, an embedding follows straight from the definitions.

Let $\omega \in \mathscr{F}(A, \mathfrak{g})$, and set $\omega^{\prime}=\bar{\varphi}(\omega)$. The map $\varphi$ then defines a map of cochain complexes, $\tilde{\varphi}:=\varphi \otimes \operatorname{id}_{V}:\left(A \otimes V, d_{\omega}\right) \rightarrow\left(A^{\prime} \otimes V, d_{\omega^{\prime}}\right)$. It is readily seen that our hypothesis on $\varphi$ is inherited by the map $\tilde{\varphi}$, as well as by the induced homomorphism in cohomology, $\tilde{\varphi}_{*}: H^{\bullet}\left(A \otimes V, d_{\omega}\right) \rightarrow$ $H^{\bullet}\left(A^{\prime} \otimes V, d_{\omega^{\prime}}\right)$. All the assertions on the resonance varieties now follow from this observation.

If we replace in the hypothesis of the above lemma the map $\varphi: A \rightarrow A^{\prime}$ by the induced homomorphism $\varphi_{*}: H^{\bullet}(A) \rightarrow H^{\bullet}\left(A^{\prime}\right)$, the conclusions may no longer follow. We illustrate this assertion with a simple example. 
Example 2.7. Let $A^{\prime} \cdot \bigwedge^{\bullet}\left(h^{*}, x^{*}\right)$ be the exterior algebra with generators in degree 1 and with differential given by $d h^{*}=0$ and $d x^{*}=x^{*} \wedge h^{*}$ (as we shall see later, this is the cochain cdga of the solvable, 2-dimensional Lie algebra $\mathfrak{s o l}_{2}$ ), and let $\varphi: A \hookrightarrow A^{\prime}$ be the inclusion of the sub-cdga $A^{\bullet}=\bigwedge^{\bullet}\left(h^{*}\right)$. We then have $H^{1}(A)=H^{1}\left(A^{\prime}\right)=\mathbb{C}$, and $\varphi_{*}$ is an isomorphism in all degrees. Nevertheless, $\mathscr{R}_{1}^{1}(A)=\{0\}$, while $\mathscr{R}_{1}^{1}\left(A^{\prime}\right)=\{0,1\}$.

Remark 2.8. The behavior illustrated in the previous example is in marked contrast with the local case. Indeed, assume $\varphi_{*}: H^{\bullet}(A) \rightarrow H^{\bullet}\left(A^{\prime}\right)$ is an isomorphism up to degree $q$ and a monomorphism in degree $q+1$. Let $\mathfrak{a}$ be an Artinian local algebra, with maximal ideal $\mathfrak{m}$. For $\omega \in \mathscr{F}(A, \mathfrak{g} \otimes \mathfrak{m})$, set $\omega^{\prime}=\varphi \otimes \operatorname{id}_{\mathfrak{g}} \otimes \operatorname{id}_{\mathfrak{a}}(\omega)$. Then, as shown in [6, Theorem 3.7], the induced homomorphism, $\tilde{\varphi}_{*}: H^{\bullet}\left(A \otimes V \otimes \mathfrak{a}, d_{\omega}\right) \rightarrow H^{\bullet}\left(A^{\prime} \otimes V \otimes \mathfrak{a}, d_{\omega^{\prime}}\right)$, inherits the properties of $\varphi_{*}$.

Returning now to the cdga map $\varphi: A \hookrightarrow A^{\prime}$ from Example 2.7, take $(\mathfrak{a}, \mathfrak{m})=(\mathbb{C}, 0)$, and consider the non-local flat connection $\omega=1 \in \mathscr{F}(A, \mathbb{C} \otimes \mathfrak{a})$. We then have $H^{1}\left(A \otimes \mathbb{C} \otimes \mathfrak{a}, d_{\omega}\right)=0$, while $H^{1}\left(A^{\prime} \otimes \mathbb{C} \otimes \mathfrak{a}, d_{\omega^{\prime}}\right) \neq 0$.

2.6. Germs of jump loci. The link between the characteristic and resonance varieties of a space is provided by a construction due to Dennis Sullivan [25].

Given a path-connected space $X$, let $\Omega^{\bullet}(X)$ be Sullivan's de Rham model of $X$, a cdga which mimics the algebra of forms on a smooth manifold, and for which the de Rham theorem holds. We say that two cdga's $A$ and $B$ have the same $q$-type, for some $q \geqslant 1$ (written $A \simeq_{q} B$ ) if they can be connected by a zig-zag of cdga maps inducing isomorphisms in cohomology in degree up to $q$, and a monomorphism in cohomology in degree $q+1$. We then have the following foundational result of Dimca and Papadima [6] (see also [7] for the case when $q=1$ and $X$ is 1-formal).

Theorem 2.9 ([6]). Let $X$ be a space of finite q-type, and assume $\Omega^{\bullet}(X)$ has the same q-type as a cdga $A$ of finite q-type. Let $\iota: G \rightarrow \mathrm{GL}(V)$ be a rational representation of a linear algebraic group $G$, and let $\theta: \mathfrak{g} \rightarrow \mathfrak{g l}(V)$ be its tangent representation.

There is then an analytic isomorphism of germs, e: $\mathscr{F}(A, \mathfrak{g})_{(0)} \stackrel{\simeq}{\longrightarrow} \operatorname{Hom}(\pi, G)_{(1)}$, which restricts to analytic isomorphisms e: $\mathscr{R}_{r}^{i}(A, \theta)_{(0)} \stackrel{\simeq}{\longrightarrow} \mathscr{V}_{r}^{i}(X, \iota)_{(1)}$, for all $i \leqslant q$ and $r \geqslant 0$.

Using this result, we now derive as an application a rather general form of the much-studied "tangent cone inclusion".

First, we need to recall a notion originally due to Body and Sullivan, see [2] and [6]. Let $A$ be a rationally defined cdga. We say $A$ has positive weights, if $A^{i}=\bigoplus_{j \in \mathbb{Z}} A_{j}^{i}$ for each degree $i \geqslant 0$, and, moreover, these vector space decompositions are compatible with the cdga structure, and satisfy the condition $A_{j}^{1}=0$, for all $j \leqslant 0$.

Theorem 2.10. Let $X$ be a space of finite q-type, and assume $\Omega^{\bullet}(X)$ has the same q-type as a cdga $A$ of finite q-type. Then:

(1) The tangent cone at 0 to the resonance variety $\mathscr{R}_{r}^{i}(A)$ is contained in the usual resonance variety $\mathscr{R}_{r}^{i}\left(H^{\bullet}(A)\right)$, for all $i \leqslant q$ and all $r \geqslant 0$.

(2) Suppose that, moreover, $A$ is defined over $\mathbb{Q}$, the identification $H^{1}\left(\Omega^{\bullet}(X)\right) \cong H^{1}(A)$ preserves $\mathbb{Q}$-structures, and $A$ has positive weights. Then $\mathscr{R}_{r}^{i}(A) \subseteq \mathscr{R}_{r}^{i}\left(H^{\bullet}(A)\right)$, for all $i \leqslant q$ and all $r \geqslant 0$.

Proof. Part (1). By [6, Theorem B], the local analytic isomorphism from Theorem 2.9 is induced by the exponential map exp: $H^{1}(X, \mathbb{C}) \rightarrow \mathbb{T}\left(\pi_{1}(X)\right)$. Hence, $\mathrm{TC}_{0}\left(\mathscr{R}_{r}^{i}(A)\right)$ is identified with $\mathrm{TC}_{1}\left(\mathscr{V}_{r}^{i}(X)\right)$. Furthermore, since $\Omega^{\bullet}(X) \simeq_{q} A$, Lemma 2.6 allows us to identify $\mathscr{R}_{r}^{i}(X)$ with $\mathscr{R}_{r}^{i}\left(H^{\bullet}(A)\right)$, for all $i \leqslant q$ and all $r$. 
On the other hand, by the finite approximation result from [20, Proposition 4.1], there is a connected, finite CW-complex $Y$ and a map $f: Y \rightarrow X$ which induces an isomorphism in cohomology up to degree $q$, and identifies the respective character tori, rank 1 characteristic varieties, and usual resonance varieties, again up to degree $q$. Finally, by a result of Libgober [14], we have that $\operatorname{TC}_{1}\left(\mathscr{V}_{r}^{i}(Y)\right) \subseteq \mathscr{R}_{r}^{i}(Y)$ for all $i$ and $r$.

Part (2). By [6, Theorem C], for each $i \leqslant q$, the variety $\mathscr{R}_{r}^{i}(A)$ is a finite union of linear subspaces of $H^{1}(A)$. Consequently, $\mathrm{TC}_{0}\left(\mathscr{R}_{r}^{i}(A)\right)=\mathscr{R}_{r}^{i}(A)$.

2.7. Spaces with $q$-finite models. As noted in [6], there are several important classes of spaces that satisfy the finiteness hypothesis of Theorems 2.9 and 2.10. Let us briefly describe the main examples of spaces $X$ which are both of finite $q$-type and admit a cdga model $A \simeq_{q} \Omega^{\bullet}(X)$ of finite $q$-type.

Example 2.11. A path-connected space $X$ is said to be $q$-formal, for some $q \geqslant 1$, if $\Omega^{\bullet}(X)$ has the same $q$-type as the cohomology ring $H^{\bullet}(X, \mathbb{C})$, endowed with the 0 differential. Evidently, if $X$ is both $q$-finite and $q$-formal, we may take $A=\left(H^{\bullet}(X, \mathbb{C}), d=0\right)$ as a suitable model for it.

We say a discrete group $\pi$ is $q$-formal if it admits a classifying space $K(\pi, 1)$ which is $q$-formal. In group-theoretic terms, $\pi$ is 1-formal if and only if the Malcev-Lie algebra of $\pi$ (in the sense of Quillen [23]) is the completion of a quadratic Lie algebra. It is readily seen that a connected CW-complex is 1-formal if and only if its fundamental group is 1-formal.

Example 2.12. Let $X$ be an irreducible, smooth quasi-projective variety-for short, a quasiprojective manifold. A finite model for $X$ (with $q=\infty$ ) can be taken to be any "Gysin model", as constructed by Morgan in [16], starting from a good compactification of $X$.

Example 2.13. A well-known construction going back to Chevalley and Eilenberg [4] associates in a functorial way to each finite-dimensional Lie algebra $\mathfrak{f}$ a "cochain" differential graded algebra, $\mathcal{C} \cdot(\mathfrak{f})=\left(\bigwedge^{\bullet}\left(\mathfrak{f}^{*}\right), d\right)$, with differential $d: \mathfrak{f}^{*} \rightarrow \mathfrak{f}^{*} \wedge \mathfrak{f}^{*}$ equal to minus the dual of the Lie bracket, and further extended to the exterior algebra by the graded Leibnitz rule.

Now suppose $X=K(\pi, 1)$ is a classifying space for a finitely generated, nilpotent group $\pi$. We then may take $A^{\bullet}=\mathcal{C} \cdot(\mathfrak{n})$, where $\mathfrak{n}$ is the (nilpotent) Malcev-Lie algebra associated to $\pi$, as in [23]. With this choice of model, Theorem 2.9 holds for an arbitrary representation $\iota: G \rightarrow \operatorname{GL}(V)$ and for all $i$ and $r$, see [6, Corollary 9.16].

The next example (a particular case of the construction described just above) shows that the inclusions from Theorem 2.10 may well be strict.

Example 2.14. Let $\pi$ be the group of integral, upper diagonal $3 \times 3$ matrices with 1 s on the diagonal. Then $\pi$ is a finitely generated, torsion-free nilpotent group, with Malcev-Lie algebra the 3 -dimensional, 2-step nilpotent Lie algebra $\mathfrak{h e i s}_{3}$. In the usual coordinates, the cochain algebra $A^{\bullet}=\mathcal{C}^{\bullet}\left(\mathfrak{h e i s}_{3}\right)$ may be presented as $A^{\bullet}=\bigwedge^{*}\left(x^{*}, y^{*}, z^{*}\right)$, with $d x^{*}=d y^{*}=0$ and $d z^{*}=x^{*} \wedge y^{*}$. Clearly, $A^{*}$ is $\infty$-finite, defined over $\mathbb{Q}$, and has positive weights $\left(1\right.$ on $x^{*}$ and $y^{*}$, and 2 on $\left.z^{*}\right)$. On the other hand, it is readily checked that $\mathscr{R}_{1}^{1}(A)=\{0\}$, while $\mathscr{R}_{1}^{1}\left(H^{\bullet}(A)\right)=\mathbb{C}^{2}$.

\section{EsSENTIALLY RANK 1 FLAT CONNECTIONS}

In this section, we single out an important subset of the parameter space for flat connections, and study its relationship with the depth-1 resonance varieties. 
3.1. Rank 1 flat connections. We start with a simple definition. As before, let $A$ be a connected cdga, and let $\mathfrak{g}$ be a Lie algebra.

Lemma 3.1. The bilinear map $P: A^{1} \times \mathfrak{g} \rightarrow A^{1} \otimes \mathfrak{g},(\eta, g) \mapsto \eta \otimes g$ induces a map

$$
P: H^{1}(A) \times \mathfrak{g} \rightarrow \mathscr{F}(A, \mathfrak{g}) .
$$

Proof. If $\eta \in Z^{1}(A)$, then clearly $\eta \otimes g$ satisfies the Maurer-Cartan equation (10). Thus, $P$ restricts to a map $P: Z^{1}(A) \times \mathfrak{g} \rightarrow \mathscr{F}(A, \mathfrak{g})$. Now, since $A$ is connected, $Z^{1}(A)=H^{1}(A)$, and so we are done.

Definition 3.2. The essentially rank one part of $\mathscr{F}(A, \mathfrak{g})$ is the set $\mathscr{F}^{1}(A, \mathfrak{g}):=P\left(H^{1}(A) \times \mathfrak{g}\right)$. We call its complement the regular part of $\mathscr{F}(A, \mathfrak{g})$.

Now suppose $\theta: \mathfrak{g} \rightarrow \mathfrak{g l}(V)$ is a finite-dimensional representation. We may then single out a subset of $\mathscr{F}^{1}(A, \mathfrak{g})$, defined as

$$
\Pi(A, \theta)=P\left(H^{1}(A) \times V(\operatorname{det} \circ \theta)\right),
$$

where det: $\mathfrak{g l}(V) \rightarrow \mathbb{C}$ is the determinant, and $V(\operatorname{det} \circ \theta)=\{g \in \mathfrak{g} \mid \operatorname{det}(\theta(g))=0\}$.

Lemma 3.3. Suppose $A$ is 1 -finite and $\mathfrak{g}$ is finite-dimensional. Then,

(1) $\mathscr{F}^{1}(A, \mathfrak{g})$ is an irreducible, Zariski-closed subset of $\mathscr{F}(A, \mathfrak{g})$ containing 0.

(2) $\Pi(A, \theta)$ is a Zariski-closed subset of $\mathscr{F}^{1}(A, \mathfrak{g})$ containing 0 .

Proof. Plainly, $\mathscr{F}^{1}(A, \mathfrak{g})$ is either $\{0\}$, or the cone on $\mathbb{P}\left(H^{1}(A)\right) \times \mathbb{P}(\mathfrak{g})$. The other claims follow at once.

The above definitions allow us to describe the 0 -th resonance variety of a cdga in a simple, yet important situation.

Lemma 3.4. Suppose $A$ is 1 -finite, $\mathfrak{g}=\mathfrak{s l}_{2}$, and $V=\mathbb{C}^{2}$ is the defining representation, given by the inclusion $\theta: \mathfrak{s l}_{2} \hookrightarrow \mathfrak{g l}_{2}$. Then $\mathscr{R}_{1}^{0}(A, \theta)=\Pi(A, \theta)$. Moreover, the variety $\mathscr{R}_{1}^{0}(A, \theta)$ is irreducible, and its dimension is positive, provided $H^{1}(A) \neq 0$.

Proof. Pick a basis $\left\{\eta_{i}\right\}$ for $A^{1}$, and let $\omega=\sum_{i} \eta_{i} \otimes g_{i}$ be a flat $\mathfrak{s l}_{2}$-connection. From Lemma 2.3, we know that $\omega$ belongs to $\mathscr{R}_{1}^{0}(A, \theta)$ if and only if $\bigcap_{i} \operatorname{ker}\left(\theta\left(g_{i}\right)\right)$ contains a non-zero vector in $\mathbb{C}^{2}$. In coordinates, this means that all matrices $g_{i}$ are of the form $\left(\begin{array}{cc}0 & \lambda_{i} \\ 0 & 0\end{array}\right)$, with $\sum_{i} \lambda_{i} \eta_{i} \in H^{1}(A)$. It follows that $\mathscr{R}_{1}^{0}(A, \theta)=\Pi(A, \theta)$, as claimed. The remaining assertions follow from the fact that $V(\operatorname{det} \circ \theta)$ is an irreducible hypersurface in $\mathfrak{s l}_{2}$.

3.2. Rank 1 flat connections and resonance. The next results describe in full generality the way in which the essentially rank 1 part of the set of flat connections, $\mathscr{F}^{1}(A, \mathfrak{g})$, cuts the depth 1 resonance varieties $\mathscr{R}_{1}^{k}(A, \theta)$. In view of Remark 2.5, we start with the "universal" case.

Theorem 3.5. Let $A$ be a connected cdga. Suppose $\mathfrak{g}=\mathfrak{g l}_{n}$ and $\theta=\mathrm{id}_{\mathfrak{g}}$, and let $\omega=\eta \otimes b$ be an arbitrary element of $\mathscr{F}^{1}(A, \mathfrak{g})$. Then $\omega$ belongs to $\mathscr{R}_{1}^{k}(A, \theta)$ if and only if there is an eigenvalue $\lambda$ of $b$ such that $\lambda \eta$ belongs to $\mathscr{R}_{1}^{k}(A)$.

Proof. We start by putting the matrix $b$ in Jordan canonical form, so that $\mathbb{C}^{n}=\bigoplus_{i} V_{i}$, where $V_{i}$ are the generalized eigenspaces of $b$, and $b=\oplus_{i} b_{i}$, where $b_{i}$ are the Jordan blocks. Setting $\omega_{i}=\eta \otimes b_{i}$, the Aomoto complex splits accordingly, and so does its cohomology,

$$
H^{k}\left(A \otimes \mathbb{C}^{n}, d_{\omega}\right)=\bigoplus_{i} H^{k}\left(A \otimes V_{i}, d_{\omega_{i}}\right) .
$$


Thus, it is enough to consider the case when $b$ is a single Jordan block, of size $n \geqslant 2$, and with eigenvalue $\lambda$.

In the standard basis $e_{1}, \ldots, e_{n}$ for $\mathbb{C}^{n}$, the differential $d_{\omega}$ is given by

$$
d_{\omega}\left(\sum_{j=1}^{n} \alpha_{j} \otimes e_{j}\right)=\sum_{j<n}\left(d \alpha_{j}+\lambda \eta \alpha_{j}+\eta \alpha_{j+1}\right) \otimes e_{j}+\left(d \alpha_{n}+\lambda \eta \alpha_{n}\right) \otimes e_{n}
$$

Let $\mathbb{C}^{n-1}$ be the subspace of $\mathbb{C}^{n}$ spanned by $e_{1}, \ldots, e_{n-1}$. Clearly, $A \otimes \mathbb{C}^{n-1}$ is a subcomplex of $A \otimes \mathbb{C}^{n}$ with respect to $d_{\omega}$. Moreover, the induced differential on the quotient complex $A \otimes \mathbb{C} \cdot e_{n}$ may be identified with the rank 1 differential $d_{\lambda \eta}: A \rightarrow A$ sending $\alpha \in A$ to $d \alpha+\lambda \eta \alpha$.

Using (20), it is readily verified that the connecting homomorphism in the associated long exact sequence in cohomology, $\delta:: H^{\bullet}\left(A \otimes \mathbb{C}, d_{\lambda \eta}\right) \rightarrow H^{\bullet+1}\left(A \otimes \mathbb{C}^{n-1}, d_{\omega}\right)$, sends the class of $\alpha \in A$ to the class of $\eta \alpha \otimes e_{n-1}$. It remains to establish the following equivalence

$$
H^{k}\left(A \otimes \mathbb{C}^{n}, d_{\omega}\right)=0 \Longleftrightarrow H^{k}\left(A \otimes \mathbb{C}, d_{\lambda \eta}\right)=0 .
$$

To prove the backwards implication, we may iterate the above construction and use the associated long exact sequences to infer that the natural map, $H^{k}\left(A \otimes \mathbb{C} \cdot e_{1}, d_{\omega}\right) \rightarrow H^{k}\left(A \otimes \mathbb{C}^{n}, d_{\omega}\right)$, is onto. On the other hand, it follows from $(20)$ that $\left(A \otimes \mathbb{C} \cdot e_{1}, d_{\omega}\right)$ can be naturally identified with $\left(A, d_{\lambda \eta}\right)$, and the claim follows.

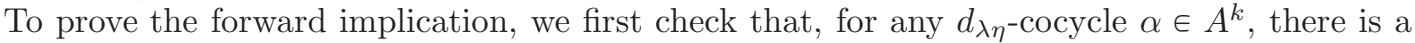
$\beta \in A^{k}$ such that $\eta \alpha+d_{\lambda \eta}(\beta)=0$. Indeed, the class of the $d_{\omega}$-cocycle $\alpha \otimes e_{1}$ in $H^{k}\left(A \otimes \mathbb{C}^{n-1}, d_{\omega}\right)$ lies in the image of $\delta_{k-1}$, by our vanishing assumption. This means that there is a $d_{\lambda \eta}$-cocycle $\beta^{\prime} \in A^{k-1}$ such that $\alpha \otimes e_{1}=\eta \beta^{\prime} \otimes e_{n-1}+\sum_{j<n} d_{\omega}\left(\beta_{j}^{\prime} \otimes e_{j}\right)$, for some $\beta_{j}^{\prime} \in A^{k-1}$. By (20) and graded-commutativity, it follows that $\eta \alpha \otimes e_{1}=\sum_{j<n} \eta d \beta_{j}^{\prime} \otimes e_{j}$. Hence,

$$
\eta \alpha=\eta d \beta_{1}^{\prime}=-d\left(\eta \beta_{1}^{\prime}\right)=d_{\lambda \eta}\left(-\eta \beta_{1}^{\prime}\right),
$$

where the second equality comes from the (graded) Leibnitz rule. Thus, we may take $\beta=\eta \beta_{1}^{\prime}$.

Now consider the element $\beta \otimes e_{1}+\alpha \otimes e_{2} \in A^{k} \otimes \mathbb{C}^{n}$, where $\alpha$ is an arbitrary $d_{\lambda \eta}$-cocycle in $A^{k}$, and $\beta$ is chosen so that $\eta \alpha+d_{\lambda \eta}(\beta)=0$. Using again (20), it is readily verified that $d_{\omega}\left(\beta \otimes e_{1}+\alpha \otimes e_{2}\right)=0$, from which we infer that $\beta \otimes e_{1}+\alpha \otimes e_{2}=d_{\omega}\left(\sum_{j=1}^{n} \beta_{j} \otimes e_{j}\right)$. Therefore, $\alpha=d_{\lambda \eta}\left(\beta_{2}\right)+\eta \beta_{3}$, where $\beta_{3}=0$ if $n=2$. It follows that $\delta_{k}$ sends the class of $\alpha$ in $H^{k}\left(A \otimes \mathbb{C}, d_{\lambda \eta}\right)$ to 0 . Hence, $\delta_{k}$ is the zero map, which implies that $H^{k}\left(A \otimes \mathbb{C}, d_{\lambda \eta}\right)=0$, since $H^{k}\left(A \otimes \mathbb{C}^{n}, d_{\omega}\right)=0$. This completes the proof.

Remark 3.6. As suggested by the referee, the above theorem can be interpreted in terms of the spectral sequence $\left(E^{k}, d^{k}\right)$ associated to the differential module $C=\left(A \otimes \mathbb{C}^{n}, d_{\omega}\right)$, endowed with the finite, increasing filtration defined by $F_{0}=0$ and $F_{s}=A \otimes \operatorname{span}\left\{e_{1}, \ldots, e_{s}\right\}$ for $1 \leqslant s \leqslant n$ and differential $d_{\omega}$ of degree 1 . The degree $q$ part of the first page of this (convergent) spectral sequence has the form $\oplus_{\lambda} \oplus_{n_{\lambda}} H^{q}\left(A, d_{\lambda \eta}\right)$, where $\lambda$ runs through the eigenvalues of the matrix $b$, and $n_{\lambda}$ is the multiplicity of $\lambda$. Theorem 3.5 can then be restated as saying:

$$
H^{q}(C)=0 \Longleftrightarrow \text { the degree } q \text { part of } E^{1} \text { vanishes. }
$$

The backwards implication is obvious, since $\operatorname{gr}_{s}\left(H^{q}(C)\right)$ is isomorphic to the degree $q$ part of $E_{s}^{\infty}$, but the forward implication is quite subtle, and cannot be proved by a simple degeneration argument. Indeed, as the next example shows, the presence of non-trivial higher Massey products in the cdga $A$ may very well yield non-trivial differentials in the $E^{2}$ page. 
Example 3.7. Let $A=(\bigwedge(x, y, z) ; d x=d y=0, d z=x y)$ be the minimal model of the Heisenberg 3-dimensional nilmanifold, and consider the spectral sequence associated to the essentially rank 1 flat connection $\omega=y \otimes b$, where $b=\left(\begin{array}{lll}0 & 1 & 0 \\ 0 & 0 & 1 \\ 0 & 0 & 0\end{array}\right)$. We claim that the differential $d^{2}: E_{3}^{2} \rightarrow E_{1}^{2}$ is non-zero.

To prove the claim, consider the (degree 1) class of $\alpha=z \otimes e_{2}+x \otimes e_{3} \in Z_{3}^{2}$ in $E_{3}^{2}$, and suppose that $d^{2}([\alpha])=0 \in E_{1}^{2}$. Our assumption implies that $d_{\omega}(\alpha) \in Z_{0}^{1}+d_{\omega} Z_{2}^{1}=d_{\omega} Z_{2}^{1}$. Hence, there is an element $\beta=\beta_{1} \otimes e_{1}+\beta_{2} \otimes e_{2}$ with $d \beta_{2}=0$ such that $d_{\omega}(\alpha)=d_{\omega}(\beta)$. Equivalently, we have $y z=d \beta_{1}+y \beta_{2}$, for some $\beta_{1} \in A^{1}$ and $\beta_{2} \in A^{1}$ with $d \beta_{2}=0$. It follows that $y z=d\left(c_{1} z\right)+y\left(c_{2} x+c_{3} y\right)$, for some constants $c_{i} \in \mathbb{C}$. This leads to the equality $y z=\left(c_{1}-c_{2}\right) x y$ in $A^{2}$, a contradiction.

We now turn to the general case, in which $A$ is a connected cdga, $\mathfrak{g}$ is an arbitrary Lie algebra, and $\theta: \mathfrak{g} \rightarrow \mathfrak{g l}(V)$ is a finite-dimensional representation.

Corollary 3.8. Let $\omega=\eta \otimes g$ be an arbitrary element of $\mathscr{F}^{1}(A, \mathfrak{g})$. Then $\omega$ belongs to $\mathscr{R}_{1}^{k}(A, \theta)$ if and only if there is an eigenvalue $\lambda$ of $\theta(g)$ such that $\lambda \eta$ belongs to $\mathscr{R}_{1}^{k}(A)$. Moreover,

$$
\Pi(A, \theta) \subseteq \bigcap_{k: H^{k}(A) \neq 0} \mathscr{R}_{1}^{k}(A, \theta) .
$$

Proof. In view of Remark 2.5, the element $\omega$ belongs to $\mathscr{R}_{1}^{k}(A, \theta)$ if and only if $\eta \otimes \theta(g)$ belongs to $\mathscr{R}_{1}^{k}\left(A, \operatorname{id}_{\mathfrak{g l}(V)}\right)$. Hence, the first claim follows from Theorem 3.5.

To prove the second claim, start by noting that $\theta(g)$ has the eigenvalue $\lambda=0$. By (15), then, $\lambda \eta \in \mathscr{R}_{1}^{k}(A)$ if and only if $H^{k}(A) \neq 0$, and this completes the proof.

3.3. Some representation theory. Next, we analyze the sets $V(\operatorname{det} \circ \theta)$ in the case when $\theta: \mathfrak{g} \rightarrow \mathfrak{g l}(V)$ is the structure map of a (non-zero) finite-dimensional module over $\mathfrak{g}=\mathfrak{s l}_{2}$. We refer the reader to $[9,12]$ for the necessary ingredients from classical representation theory.

Let $\{H, X, Y\}$ be the standard basis of $\mathfrak{s l}_{2}$, and let $\mathfrak{t}=\mathbb{C} \cdot H$ be the standard Cartan subalgebra. The representation $V$ decomposes as a direct sum of irreducible representations of the form $V(n)$, with $n \geqslant 0$, where $\operatorname{dim} V(n)=n+1$, and the eigenvalues of $H$ on $V(n)$ are $n, n-2, \ldots,-n$. We denote the structure map of $V(n)$ by $\theta_{n}$. The defining representation of $\mathfrak{s l}_{2}$ is $V(1)$, while $\theta_{1}$ is the inclusion $\mathfrak{s l}_{2} \hookrightarrow \mathfrak{g l}_{2}$. Finally, we denote the map det $\circ \theta_{1}: \mathfrak{s l}_{2} \rightarrow \mathbb{C}$ simply by det.

Lemma 3.9. If $V$ has a direct summand equal to $V(n)$, with $n$ even, then $V(\operatorname{det} \circ \theta)=\mathfrak{s l}_{2}$. Otherwise, $V(\operatorname{det} \circ \theta)=V(\operatorname{det})$.

Proof. It is enough to check that det $\circ \theta_{n}$ is equal to 0 if $n$ is even, and is a non-zero multiple of $\operatorname{det}^{(n+1) / 2}$ if $n$ is odd. In order to verify this assertion, we shall use some basic invariant theory.

Let $\iota: \mathrm{SL}_{2} \rightarrow \mathrm{GL}(V)$ be the (unique) rational representation for which $d_{1}(\iota)=\theta$. We first claim that det $\circ \theta$ belongs to $\mathbb{C}\left[\mathfrak{s l}_{2}^{*}\right]^{\mathrm{SL}_{2}}$, the invariant subalgebra of the polynomial algebra on the dual vector space to $\mathfrak{s l}_{2}$, taken with respect to the adjoint representation of $\mathrm{SL}_{2}$. Indeed, note that $\iota \circ c(s)=c(\iota(s)) \circ \iota$, for any $s \in \mathrm{SL}_{2}$, where $c(-)$ stands for the conjugation action. The claim then follows upon taking differential at 1.

Next, let $W=\mathbb{Z}_{2}$ be the Weyl group of $\mathrm{SL}_{2}$, acting on $\mathfrak{t}$ by the alternating representation, and consider the natural morphism $r: \mathbb{C}\left[\mathfrak{s l}_{2}^{*}\right]^{\mathrm{SL}_{2}} \rightarrow \mathbb{C}\left[\mathfrak{t}^{*}\right]^{W}$ between the respective subalgebras of invariants. Clearly, $r$ is surjective, since $\mathbb{C}\left[\mathfrak{t}^{*}\right]^{W}=\mathbb{C}\left[\left(H^{*}\right)^{2}\right]$ and $r($ det $)=-\left(H^{*}\right)^{2}$. Furthermore, $r$ is also injective, since every element of $\mathfrak{s l}_{2}$ outside of $V$ (det) is $\mathrm{SL}_{2}$-conjugate to an element of t. Thus, $\mathbb{C}\left[\mathfrak{s l}_{2}^{*}\right]^{\mathrm{SL}_{2}}=\mathbb{C}[$ det $]$. 
Finally, assume $V=V(n)$. Since $\operatorname{deg}\left(\operatorname{det} \circ \theta_{n}\right)=n+1$, we must have $\operatorname{det} \circ \theta_{n}=0$ if $n$ is even, and $\operatorname{det} \circ \theta_{n}=\lambda_{n} \operatorname{det}^{(n+1) / 2}$ if $n$ is odd. But $\operatorname{det}\left(\theta_{n}(H)\right) \neq 0$, and so $\lambda_{n} \neq 0$, thus completing the proof.

\section{Holonomy Lie algebras of Differential graded algebras}

Our primary aim in this section is to extend the definition of holonomy Lie algebras to arbitrary cdga's, and to extend several results from [7], thereby relating flat connections to Lie algebra representations and resonance varieties of cdga's to resonance varieties of Lie algebras. As a first application, we compute the depth-1 resonance varieties of finite-dimensional, nilpotent Lie algebras, in two important non-abelian cases.

4.1. Holonomy Lie algebra. Let $A^{\bullet}$ be a connected cdga. For our purposes here, we will be mainly interested in the differential $d: A^{1} \rightarrow A^{2}$ and the product $\cup: A^{1} \wedge A^{1} \rightarrow A^{2}$. Our starting point is the following lemma, whose proof is straightforward.

Lemma 4.1. For any representation $\theta: \mathfrak{g} \rightarrow \mathfrak{g l}(V)$, both the parameter space $\mathscr{F}(A, \mathfrak{g})$ and the resonance varieties $\mathscr{R}_{r}^{0}(A, \theta)$ and $\mathscr{R}_{r}^{1}(A, \theta)$ depend only on the co-restriction of $d$ and $\cup$ to the subspace $\operatorname{im}(d)+\operatorname{im}(\cup) \subseteq A^{2}$.

This leads us to the following construction. Given a $\mathbb{C}$-vector space $W$, denote by $\mathbf{L} \cdot(W)$ the free Lie algebra on $W$, graded by bracket length. In low degrees, $\mathbf{L}^{1}(W)=W$, while $\mathbf{L}^{2}(W)$ may be identified with $W \wedge W$ via $[u, v] \leftrightarrow u \wedge v$.

Now let $A$ be a 1-finite cdga. Set $A_{i}=\left(A^{i}\right)^{*}$, and let $\mathbf{L}\left(A_{1}\right)$ be the free Lie algebra on the dual vector space $A_{1}$. We then have dual maps, $d^{*}: A_{2} \rightarrow A_{1}=\mathbf{L}^{1}\left(A_{1}\right)$ and $\cup^{*}: A_{2} \rightarrow A_{1} \wedge A_{1}=$ $\mathbf{L}^{2}\left(A_{1}\right)$.

Definition 4.2. The holonomy Lie algebra of a 1-finite cdga $A$ is the finitely presented Lie algebra $\mathfrak{h}(A)=\mathbf{L}\left(A_{1}\right) / \operatorname{ideal}\left(\operatorname{im} \partial_{A}\right)$, where $\partial_{A}$ is the linear map

$$
\partial_{A}:=d^{*}+\cup^{*}: A_{2} \rightarrow \mathbf{L}^{1}\left(A_{1}\right) \oplus \mathbf{L}^{2}\left(A_{1}\right) \subset \mathbf{L}\left(A_{1}\right) .
$$

This construction is functorial. Indeed, if $\varphi: A \rightarrow A^{\prime}$ is a cdga map, then the linear map $\varphi_{1}=\left(\varphi^{1}\right)^{*}: A_{1}^{\prime} \rightarrow A_{1}$ extends to Lie algebra map $\mathbf{L}\left(\varphi_{1}\right): \mathbf{L}\left(A_{1}^{\prime}\right) \rightarrow \mathbf{L}\left(A_{1}\right)$, which in turn induces a Lie algebra map $\mathfrak{h}(\varphi): \mathfrak{h}\left(A^{\prime}\right) \rightarrow \mathfrak{h}(A)$.

Remark 4.3. In the case when $d=0$, the above definition recovers the classical definition of the holonomy Lie algebra of an algebra, due to K. T. Chen [3]. In this situation, $\mathfrak{h}(A)$ inherits a natural grading from the free Lie algebra, compatible with the Lie bracket; thus, $\mathfrak{h}(A)$ is a finitely presented graded Lie algebra, with generators in degree 1 , and relations in degree 2 . In general, though, the ideal generated by $\operatorname{im}\left(\partial_{A}\right)$ is not homogeneous, and the Lie algebra $\mathfrak{h}(A)$ is not graded.

Example 4.4. Let $\mathfrak{f}$ be a finite-dimensional Lie algebra, and let $A^{\bullet}=\mathcal{C} \cdot(\mathfrak{f})$ be its cochain cdga, as in Example 2.13. Then $\mathfrak{h}(A)$ is the quotient of $\mathbf{L}(\mathfrak{f})$ by all the relations of the form $[x, y]_{\mathfrak{f}}=[x, y]_{\mathbf{L}(\mathfrak{f})}$, with $x, y \in \mathfrak{f}$. Hence, $\mathfrak{h}(\mathcal{C} \cdot(\mathfrak{f}))=\mathfrak{f}$, as (ungraded) Lie algebras. 
4.2. Flat connections and representations. We now relate the set of flat connections on a cdga with the set of Lie algebra representations of the corresponding holonomy Lie algebra.

To this end, let us start with an arbitrary finitely-generated Lie algebra $\mathfrak{h}$, presented as the quotient $\mathbf{L}\left(A_{1}\right) /$ ideal $(\operatorname{im} \partial)$ of the free Lie algebra on a finite-dimensional vector space $A_{1}$ by the ideal generated by the image of a linear map, $\partial: A_{2} \rightarrow \mathbf{L}\left(A_{1}\right)$.

Now let $\mathfrak{g}$ be a Lie algebra, and let $\operatorname{Hom}_{\text {Lie }}(\mathfrak{h}, \mathfrak{g})$ be the set of Lie algebra morphisms from $\mathfrak{h}$ to $\mathfrak{g}$. Every such morphism defines by restriction a linear map from $A_{1}$ to $\mathfrak{g}$. Thus, we have a canonical inclusion,

$$
\operatorname{Hom}_{\text {Lie }}(\mathfrak{h}, \mathfrak{g}) \subseteq \operatorname{Hom}\left(A_{1}, \mathfrak{g}\right) .
$$

We let $\operatorname{Hom}_{\text {Lie }}^{1}(\mathfrak{h}, \mathfrak{g})$ denote the intersection of $\operatorname{Hom}_{\text {Lie }}(\mathfrak{h}, \mathfrak{g})$ with $\operatorname{Hom}^{1}\left(A_{1}, \mathfrak{g}\right)$. When $\mathfrak{g}$ is finite-dimensional, it is readily checked that $\operatorname{Hom}_{\text {Lie }}(\mathfrak{h}, \mathfrak{g})$ is a Zariski-closed subset of the affine space $\operatorname{Hom}\left(A_{1}, \mathfrak{g}\right)$. This endows the representation variety $\operatorname{Hom}_{\text {Lie }}(\mathfrak{h}, \mathfrak{g})$ with an affine structure, natural in both $\mathfrak{h}$ and $\mathfrak{g}$.

The next result extends Lemma 3.13 from [7], where the case in which $A$ has zero differential was analyzed.

Proposition 4.5. Let $A$ be a 1-finite cdga, and let $\mathfrak{g}$ be a Lie algebra. Then, the canonical isomorphism $\psi: A^{1} \otimes \mathfrak{g} \stackrel{\simeq}{\longrightarrow} \operatorname{Hom}\left(A_{1}, \mathfrak{g}\right)$ restricts to an identification

$$
\psi: \mathscr{F}(A, \mathfrak{g}) \stackrel{\simeq}{\longrightarrow} \operatorname{Hom}_{\text {Lie }}(\mathfrak{h}(A), \mathfrak{g}) .
$$

Moreover, $\psi$ further restricts to an identification

$$
\psi: \mathscr{F}^{1}(A, \mathfrak{g}) \stackrel{\simeq}{\longrightarrow} \operatorname{Hom}_{\text {Lie }}^{1}(\mathfrak{h}(A), \mathfrak{g}) .
$$

Proof. To prove the first claim, we need to show the following: an element $\omega \in A^{1} \otimes \mathfrak{g}$ satisfies the Maurer-Cartan equation (10) if and only if the extension of the map $\rho=\psi(\omega)$ to the free Lie algebra, $\rho: \mathbf{L}\left(A_{1}\right) \rightarrow \mathfrak{g}$, vanishes on $\partial_{A}(a)$, for all $a \in A_{2}$.

To that end, pick a basis $\left\{\eta_{i}\right\}$ for $A^{1}$ and denote by $\left\{\eta_{i}^{*}\right\}$ the dual basis for $A_{1}$. Writing $\omega=\sum_{i} \eta_{i} \otimes g_{i}$, we have that $\rho\left(\eta_{i}^{*}\right)=g_{i}$. In the chosen bases, the linear maps $d^{*}: A_{2} \rightarrow A_{1}$ and $\cup^{*}: A_{2} \rightarrow A_{1} \wedge A_{1}$ may be written as $d^{*}(a)=\sum_{i} d_{i} \eta_{i}^{*}$ and $\cup^{*}(a)=\sum_{i<j} \mu_{i j} \eta_{i}^{*} \wedge \eta_{j}^{*}$. Recalling that $\partial_{A}=d^{*}+\cup^{*}$, we find that

$$
\rho \partial_{A}(a)=\sum_{i} d_{i} g_{i}+\sum_{i<j} \mu_{i j}\left[g_{i}, g_{j}\right] .
$$

In the same bases, the Maurer-Cartan equation for $\omega$ can be written as (11). Applying the (injective) linear map $\psi$ to both sides of this equation, we find that (11) is equivalent to

$$
\sum_{i}\left\langle d \eta_{i}, a\right\rangle g_{i}+\sum_{i<j}\left\langle\cup\left(\eta_{i} \wedge \eta_{j}\right), a\right\rangle\left[g_{i}, g_{j}\right]=0,
$$

for all $a \in A_{2}$. Clearly, the right side of (28) equals the left side of (29), and so the first claim is proved.

As for the second claim, we need to show that $\omega$ belongs to $\mathscr{F}^{1}(A, \mathfrak{g})$, that is, $\omega$ can be written as $\eta \otimes g$, for some $\eta \in A^{1}$ and $g \in \mathfrak{g}$, if and only if $\rho=\psi(\omega)$ belongs to $\operatorname{Hom}^{1}\left(A_{1}, \mathfrak{g}\right)$, that is, $\operatorname{im}(\rho)$ has dimension at most 1 . But this is clear, and we are done. 
4.3. Lie algebra cohomology. Next, we relate the cohomology in low degrees of the holonomy Lie algebra to the cohomology of the corresponding Aomoto complex. For basic facts about cohomology of Lie algebras, we refer to the book by Hilton and Stammbach [11].

We start with an arbitrary Lie algebra $\mathfrak{h}$, presented as $\mathfrak{h}=\mathbf{L}\left(A_{1}\right) / \operatorname{ideal}\left(\operatorname{im} \partial: A_{2} \rightarrow \mathbf{L}\left(A_{1}\right)\right.$ ), and with an arbitrary h-module $V=V_{\rho}$, with structure map $\rho: \mathfrak{h} \rightarrow \mathfrak{g l}(V)$. Let $\operatorname{Der}(\mathfrak{h}, V)$ be the vector space consisting of all linear maps $\delta: \mathfrak{h} \rightarrow V$ satisfying $\delta([g, h])=\rho(g) \cdot \delta(h)-\rho(h)$. $\delta(g)$. Let $\pi: \mathbf{L}\left(A_{1}\right) \rightarrow \mathfrak{h}$ be the canonical projection; restricting to the free generators identifies $\operatorname{Der}\left(\mathbf{L}\left(A_{1}\right), V_{\rho \pi}\right)$ with $\operatorname{Hom}\left(A_{1}, V\right)$.

Let $d_{\rho}^{1}: \operatorname{Hom}\left(A_{1}, V\right) \rightarrow \operatorname{Hom}\left(A_{2}, V\right)$ be the linear map sending $\delta$ to $\delta \circ \partial$; the kernel of this map can be identified with the space $\operatorname{Der}(\mathfrak{h}, V)$. Set $A_{0}=\mathbb{C}$, and denote by $d_{\rho}^{0}: \operatorname{Hom}\left(A_{0}, V\right) \rightarrow$ $\operatorname{Hom}\left(A_{1}, V\right)$ the linear map sending $v \in V$ to the inner derivation $\delta_{v}$, defined by $\delta_{v}(h)=\rho h(v)$, for $h \in \mathfrak{h}$. Then, the cochain complex

$$
0 \longrightarrow \operatorname{Hom}\left(A_{0}, V\right) \stackrel{d_{\rho}^{0}}{\longrightarrow} \operatorname{Hom}\left(A_{1}, V\right) \stackrel{d_{\rho}^{1}}{\longrightarrow} \operatorname{Hom}\left(A_{2}, V\right) \longrightarrow 0 \longrightarrow \cdots
$$

computes the cohomology groups $H^{i}(\mathfrak{h}, V)$, for $i=0$ and 1 .

Now let $A$ be a 1-finite cdga, and let $\mathfrak{h}=\mathfrak{h}(A)$ be its holonomy Lie algebra. Consider the Lie algebra $\mathfrak{g}=\mathfrak{g l}(V)$, endowed with the representation $\theta=\operatorname{id}_{\mathfrak{g l}(V)}$, and let $\psi: \mathscr{F}(A, \mathfrak{g l}(V)) \stackrel{\simeq}{\longrightarrow}$ $\operatorname{Hom}_{\text {Lie }}(\mathfrak{h}(A), \mathfrak{g l}(V))$ be the isomorphism provided by Proposition 4.5.

Lemma 4.6. With notation as above, let $\omega \in \mathscr{F}(A, \mathfrak{g l}(V))$ be a flat connection, and let $\rho=\psi(\omega)$ be the corresponding structure map for the $\mathfrak{h}(A)$-module $V=V_{\rho}$. There is then an isomorphism $H^{i}\left(A \otimes V, d_{\omega}\right) \cong H^{i}\left(\mathfrak{h}(A), V_{\rho}\right)$ for $i=0$ and 1 .

Proof. Consider the Aomoto complex (12) and the cochain complex (30). It is readily seen that, in the range $i \leqslant 1$, the natural monomorphism $\psi: A^{i} \otimes V \rightarrow \operatorname{Hom}\left(A_{i}, V\right)$ is an isomorphism, and commutes with the differentials $d_{\omega}^{i}$ and $d_{\rho}^{i}$. The conclusion follows at once.

4.4. Resonance varieties of a Lie algebra. We now consider the Lie analogue of the resonance varieties, following the approach from [7]. Let $\mathfrak{h}$ be a Lie algebra, and let $\theta: \mathfrak{g} \rightarrow \mathfrak{g l}(V)$ be a representation of another Lie algebra. Associated to these data we have the resonance varieties

$$
\mathscr{R}_{r}^{i}(\mathfrak{h}, \theta)=\left\{\rho \in \operatorname{Hom}_{\text {Lie }}(\mathfrak{h}, \mathfrak{g}) \mid \operatorname{dim}_{\mathbb{C}} H^{i}\left(\mathfrak{h}, V_{\theta \rho}\right) \geqslant r\right\} .
$$

Lemma 4.7. Suppose $\mathfrak{h}$ is a finitely generated Lie algebra and $\theta: \mathfrak{g} \rightarrow \mathfrak{g l}(V)$ is a finite-dimensional representation of a finite-dimensional Lie algebra. Then the resonance varieties $\mathscr{R}_{r}^{i}(\mathfrak{h}, \theta)$ are Zariski-closed subsets of $\mathrm{Hom}_{\text {Lie }}(\mathfrak{h}, \mathfrak{g})$, for $i \leqslant 1$ and $r \geqslant 0$.

Proof. Let $\rho \in \operatorname{Hom}_{\text {Lie }}(\mathfrak{h}, \mathfrak{g})$, and consider the cochain complex (30), with $\rho$ replaced by $\theta \circ \rho$. It is easy to check that the differentials $d_{\theta \rho}^{i}$ depend algebraically on $\rho$. The claim then follows from [6, Lemma 9.2].

The next result generalizes Corollary 3.18 from [7], where only cdga's with differential $d=0$ were considered.

Corollary 4.8. Given a 1 -finite cdga $A$ and a Lie algebra representation $\theta: \mathfrak{g} \rightarrow \mathfrak{g l}(V)$, the canonical isomorphism $\psi: \mathscr{F}(A, \mathfrak{g}) \stackrel{\simeq}{\longrightarrow} \operatorname{Hom}_{\text {Lie }}(\mathfrak{h}(A), \mathfrak{g})$ restricts to an identification

$$
\mathscr{R}_{r}^{i}(A, \theta) \stackrel{\simeq}{\longrightarrow} \mathscr{R}_{r}^{i}(\mathfrak{h}(A), \theta),
$$

for each $i \leqslant 1$ and $r \geqslant 0$. 
Proof. Let $\omega \in \mathscr{F}(A, \mathfrak{g})$ be a flat connection, and let $\rho=\psi(\omega): \mathfrak{h}(A) \rightarrow \mathfrak{g}$ be the corresponding Lie algebra morphism. Then $\omega^{\prime}=\left(\operatorname{id}_{A^{1}} \otimes \theta\right)(\omega) \in \mathscr{F}(A, \mathfrak{g l}(V))$ corresponds to $\theta \circ \rho: \mathfrak{h}(A) \rightarrow \mathfrak{g l}(V)$, and so Lemma 4.6 provides us with an isomorphism $H^{i}\left(A \otimes V, d_{\omega^{\prime}}\right) \cong H^{i}\left(\mathfrak{h}(A), V_{\theta \rho}\right)$, for $i=0$ and 1. The desired conclusion now follows from Remark 2.5.

4.5. Finite-dimensional Lie algebras. We now consider in more detail the case when $\mathfrak{f}$ is a Lie algebra of finite dimension. Let $V_{\rho}$ be an arbitrary $\mathfrak{f}$-module. Using the standard f-resolution of $\mathbb{C}$, we see that $H^{\bullet}\left(\mathfrak{f}, V_{\rho}\right)=H^{\bullet}\left(\mathcal{C}(\mathfrak{f}) \otimes V, d_{\rho}\right)$.

Lemma 4.9. Given a finite-dimensional Lie algebra $\mathfrak{f}$ and a representation $\theta: \mathfrak{g} \rightarrow \mathfrak{g l}(V)$, let $\omega \in \mathscr{F}(\mathcal{C}(\mathfrak{f}), \mathfrak{g})$ correspond to $\rho \in \operatorname{Hom}_{\text {Lie }}(\mathfrak{f}, \mathfrak{g})$ under the isomorphism $\psi$ from Proposition 4.5. Then $d_{\theta \circ \rho}=d_{\omega}$.

Proof. Straightforward direct computation.

Corollary 4.10. For a finite-dimensional Lie algebra $\mathfrak{f}$ and a representation $\theta: \mathfrak{g} \rightarrow \mathfrak{g l}(V)$, the canonical isomorphism $\psi: \mathscr{F}(\mathcal{C}(\mathfrak{f}), \mathfrak{g}) \stackrel{\simeq}{\longrightarrow} \operatorname{Hom}_{\text {Lie }}(\mathfrak{f}, \mathfrak{g})$ identifies $\mathscr{R}_{r}^{i}(\mathcal{C}(\mathfrak{f}), \theta)$ with $\mathscr{R}_{r}^{i}(\mathfrak{f}, \theta)$, for all $i, r \geqslant 0$.

Note also that, if both $V$ and $\mathfrak{g}$ are finite-dimensional, then $\mathscr{R}_{r}^{i}(\mathfrak{f}, \theta)$ is a Zariski-closed subset of $\operatorname{Hom}_{\text {Lie }}(\mathfrak{f}, \mathfrak{g})$, for all $i, r \geqslant 0$.

4.6. Lie algebras and rank one resonance. We now revisit the ubiquitous Lie algebra $\mathfrak{s l}_{2}$. We denote by $\mathfrak{s o l}_{2}$ its standard Borel subalgebra, generated by $H$ and $X$. This 2-dimensional solvable Lie algebra is the unique nonabelian Lie algebra in dimension 2. Setting $h=\frac{1}{2} H$ and $x=X$, we find that $\mathcal{C} \cdot\left(\mathfrak{s o l}_{2}\right)=\bigwedge^{\bullet}\left(h^{*}, x^{*}\right)$ with $d h^{*}=0$ and $d x^{*}=x^{*} \wedge h^{*}$.

Remark 4.11. Later on, we will need the following easily verified fact. Suppose $\mathfrak{g}=\mathfrak{s l}_{2}$ or $\mathfrak{s o l}_{2}$, and $g, g^{\prime} \in \mathfrak{g}$; then $\left[g, g^{\prime}\right]=0$ if and only if $\operatorname{rank}\left\{g, g^{\prime}\right\} \leqslant 1$.

As shown in the next proposition, non-triviality of rank 1 resonance is detected by the Lie algebras $\mathfrak{s o l}_{2}$ and $\mathfrak{h e i s}_{3}$.

Proposition 4.12. Let $A \cdot$ be a 1-finite cdga. Then:

(1) $\mathscr{R}_{1}^{1}(A) \nsubseteq\{0\}$ if and only if $\operatorname{Hom}_{\text {Lie }}\left(\mathfrak{h}(A), \mathfrak{s o l}_{2}\right)$ contains a surjection.

(2) $\mathscr{R}_{1}^{1}\left(H^{\bullet}(A)\right) \nsubseteq\{0\}$ if and only if $\operatorname{Hom}_{\text {Lie }}\left(\mathfrak{h}(A), \mathfrak{h e i s}_{3}\right)$ contains a surjection.

Proof. By definition of resonance, the condition that $\mathscr{R}_{1}^{1}(A) \nsubseteq\{0\}$ is equivalent to the existence of elements $\alpha, \beta \in A^{1}$ with $\operatorname{rank}\{\alpha, \beta\}=2, d \alpha=0$, and $d \beta+\alpha \beta=0$. By the above description of $\mathcal{C} \cdot\left(\mathfrak{s o l}_{2}\right)$, this is equivalent to the existence of a cdga morphism $\phi: \mathcal{C} \cdot\left(\mathfrak{s o l}_{2}\right) \rightarrow A^{\bullet}$ such that $\phi^{1}$ is injective.

Likewise, the condition that $\mathscr{R}_{1}^{1}\left(H^{\bullet}(A)\right) \nsubseteq\{0\}$ means that there exist $\alpha, \beta, \gamma \in A^{1}$ such that $\operatorname{rank}\{\alpha, \beta\}=2, d \alpha=d \beta=0$, and $\alpha \beta=d \gamma$, or, equivalently, there exists a cdga morphism $\phi: \mathcal{C} \cdot\left(\mathfrak{h e i s}_{3}\right) \rightarrow A^{\bullet}$ such that $H^{1}(\phi)$ is injective.

Now let $\mathfrak{f}$ be a finite-dimensional Lie algebra. View $\operatorname{Hom}_{\mathrm{cdga}}\left(\mathcal{C}^{\bullet}(\mathfrak{f}), A^{\bullet}\right)$ inside $\operatorname{Hom}\left(\mathfrak{f}^{*}, A^{1}\right)$ and $\operatorname{Hom}_{\text {Lie }}(\mathfrak{h}(A), \mathfrak{f})$ inside $\operatorname{Hom}\left(A_{1}, \mathfrak{f}\right)$, and consider the natural identification $\operatorname{Hom}\left(\mathfrak{f}^{*}, A^{1}\right) \stackrel{\sim}{\longleftrightarrow}$ $\operatorname{Hom}\left(A_{1}, \mathfrak{f}\right)$, given by taking the transpose of matrices. Lemma 3.4 from [6] and Proposition 4.5 together imply that $\operatorname{Hom}_{\text {cdga }}\left(\mathcal{C}(\mathfrak{f}), A^{\bullet}\right)$ is identified in this way with $\operatorname{Hom}_{\text {Lie }}(\mathfrak{h}(A), \mathfrak{f})$. In both cases under consideration, denote by $\rho$ the morphism of Lie algebras corresponding to $\phi$. 
In the first case, the injectivity condition translates into the surjectivity of the restriction of $\rho: \mathfrak{h}(A) \rightarrow \mathfrak{s o l}_{2}$ to the generating set $A_{1}$ for the holonomy Lie algebra $\mathfrak{h}(A)$. Since $\mathfrak{s o l}_{2}$ has dimension 2 , this last condition is equivalent to the surjectivity of $\rho$, as claimed.

In the second case, let ab: $\mathfrak{h e i s}_{3} \rightarrow \mathbb{C}^{2}$ be the abelianization map. We may then rephrase the injectivity of $H^{1}(\phi)$ as the surjectivity of the restriction of ab $\circ \rho$ to $A_{1}$. Since the 3 -dimensional Heisenberg Lie algebra $\mathfrak{h e i s}_{3}$ is nilpotent, and since a Lie morphism between nilpotent Lie algebras is onto if and only if its abelianization is onto, this last condition is again equivalent to the surjectivity of $\rho$, as claimed.

Remark 4.13. In general, there is no implication between the vanishing resonance properties (1) and (2) from Proposition 4.12. For instance, if $A^{\bullet}=\mathcal{C}^{\bullet}\left(\mathfrak{s o l}_{2}\right)$, then $H^{1}(A)=\mathbb{C}$ and $\mathscr{R}_{1}^{1}(A)=$ $\{0,1\}$, while $\mathscr{R}_{1}^{1}\left(H^{\bullet}(A)\right)=\{0\}$. On the other hand, if $A^{\bullet}=\mathcal{C} \cdot\left(\mathfrak{h e i s}_{3}\right)$, then $H^{1}(A)=\mathbb{C}^{2}$ and $\mathscr{R}_{1}^{1}(A)=\{0\}$, while $\mathscr{R}_{1}^{1}\left(H^{\bullet}(A)\right)=\mathbb{C}^{2}$. Nevertheless, it follows from Theorem 2.10(2) that, under certain additional assumptions, one such implication holds.

4.7. Nilpotent Lie algebras. To conclude this section, we compute the germs of the depth 1 characteristic varieties of a finitely-generated nilpotent group $\pi$, relative to an arbitrary rational representation of a Lie group $G$, where $G$ is either semisimple of rank 1, or one of the Borel subgroups of such a group. As explained in Example 2.13, such a computation follows from the global computation of depth 1 resonance varieties of the cochain algebra of the corresponding finite-dimensional, nilpotent Lie algebra $\mathfrak{n}$, relative to a representation of a Lie algebra $\mathfrak{g}$, where $\mathfrak{g}$ is either $\mathfrak{s l}_{2}$ or $\mathfrak{s o l}_{2}$.

Lemma 4.14. Let $\mathfrak{n}$ be a finite-dimensional, nilpotent Lie algebra, and let $\mathfrak{g}=\mathfrak{s l}_{2}$ or $\mathfrak{g}=\mathfrak{s o l}_{2}$. Then $\mathscr{F}(\mathcal{C} \cdot(\mathfrak{n}), \mathfrak{g})=\mathscr{F}^{1}(\mathcal{C} \cdot(\mathfrak{n}), \mathfrak{g})$.

Proof. In view of Proposition 4.5 and Example 4.4, we need to show that any representation $\rho: \mathfrak{n} \rightarrow \mathfrak{g}$ satisfies $\operatorname{dim}(\operatorname{im} \rho) \leqslant 1$. Pick generators $a_{1}, \ldots, a_{n}$ for $\mathfrak{n}$. Nilpotency of $\mathfrak{n}$ guarantees the existence of an integer $k$ such that all length $k$ brackets of $\rho\left(a_{1}\right), \ldots, \rho\left(a_{n}\right)$ vanish in $\mathfrak{g}$.

Recall from Remark 4.11 that $\left[b, b^{\prime}\right]=0$ in $\mathfrak{g}$ if and only if $\operatorname{rank}\left\{b, b^{\prime}\right\} \leqslant 1$. Assuming $k>2$, we claim that the above vanishing property also holds for $k-1$. Putting together these two facts, it follows that $\operatorname{im}(\rho)$ is at most 1-dimensional, as needed.

To verify our claim, let us assume that there is a non-trivial bracket $b$ on $\rho\left(a_{1}\right), \ldots, \rho\left(a_{n}\right)$ of length $k-1$. By our assumption, $\left[\rho\left(a_{i}\right), b\right]=0$, for all $i$. Hence, $\rho\left(a_{i}\right) \in \mathbb{C} \cdot b$, for all $i$, and consequently $b=0$, since $k>2$. This contradiction completes the proof.

Note that the conclusions of the lemma may fail if $\mathfrak{n}$ is only assumed to be solvable. For instance, if $\mathfrak{n}=\mathfrak{s o l}_{2}$, then $\mathscr{F}(\mathcal{C} \cdot(\mathfrak{n}), \mathfrak{n})=\operatorname{Hom}_{\text {Lie }}(\mathfrak{n}, \mathfrak{n})$ contains the identity map.

Theorem 4.15. Let $\pi$ be a finitely generated, nilpotent group, with Malcev-Lie algebra $\mathfrak{n}$, and let $V_{\theta}$ be a finite-dimensional $\mathfrak{g}$-module, where $\mathfrak{g}=\mathfrak{s l}_{2}$ or $\mathfrak{s o l}_{2}$. Then

$$
\mathscr{R}_{1}^{k}(\mathcal{C} \cdot(\mathfrak{n}), \theta)= \begin{cases}\varnothing & \text { if } k>\operatorname{dim} \mathfrak{n}, \\ \Pi(\mathcal{C} \cdot(\mathfrak{n}), \theta) \neq \varnothing & \text { if } k \leqslant \operatorname{dim} \mathfrak{n} .\end{cases}
$$

Proof. Set $A^{\bullet}=\mathcal{C} \cdot(\mathfrak{n})$. The first assertion is now immediate, since $\mathcal{C}^{k}(\mathfrak{n})=0$ for $k>\operatorname{dim} \mathfrak{n}$.

Using the identification from Corollary 4.10 in the case when $\theta=\mathrm{id}_{\mathbb{C}}$, work of Dixmier [8] shows that $\mathscr{R}_{1}^{k}(A) \subseteq\{0\}$ for all $k$, by [8, Theorem 1$]$, and $0 \in \mathscr{R}_{1}^{k}(A)$ for $k \leqslant \operatorname{dim} \mathfrak{n}$, by $[8$, Theorem 2]. 
Now, by Lemma 4.14 , the set $\mathscr{F}(A, \mathfrak{g})$ consists of all elements of the form $\omega=\eta \otimes b$, with $\eta \in H^{1}(A)$ and $b \in \mathfrak{g}$. On the other hand, by Corollary 3.8, $\omega \in \mathscr{R}_{1}^{k}(A, \theta)$ if and only if $\lambda \eta \in \mathscr{R}_{1}^{k}(A)$, for some eigenvalue $\lambda$ of $\theta(b)$. If $k \leqslant \operatorname{dim} \mathfrak{n}$, this happens precisely when either $\eta=0$ or $\lambda=0$, i.e., when $\omega \in \Pi(A, \theta)$.

Remark 4.16. It is possible to state a more general version of Theorem 4.15, based on the aforementioned results of Dixmier. Indeed, let $\mathfrak{f}$ be an arbitrary finite-dimensional Lie algebra, and recall that we have a canonical isomorphism $\mathscr{F}(\mathcal{C} \cdot(\mathfrak{f}), \mathfrak{g l}(V)) \stackrel{\simeq}{\longrightarrow} \operatorname{Hom}_{\text {Lie }}(\mathfrak{f}, \mathfrak{g l}(V))$, which identifies a flat connection $\omega$ with the $\mathfrak{f}$-module $V_{\omega}$. Applying Lemma 4.9 to the representation $\theta=\operatorname{id}_{\mathfrak{g r}(V)}$, we see that the Aomoto complex $\left(\mathcal{C} \cdot(\mathfrak{f}) \otimes V, d_{\omega}\right)$ is naturally isomorphic to the Chevalley-Eilenberg complex $\mathcal{C} \cdot\left(\mathfrak{f}, V_{\omega}\right)$, which computes the Lie algebra cohomology of $\mathfrak{f}$ with coefficients in $V_{\omega}$. Consequently, $\mathscr{R}_{1}\left(\mathcal{C} \cdot(\mathfrak{f}), \mathrm{id}_{\mathfrak{g r}(V)}\right)$ may be identified with the set of $\mathfrak{f}$-module structures on $V$ for which $H^{\bullet}(\mathfrak{f}, V) \neq 0$.

When $\mathfrak{f}=\mathfrak{n}$ is a nilpotent Lie algebra, Theorems 1 and 2 from [8] imply that $\mathscr{R}_{1}^{k}\left(\mathcal{C} \cdot(\mathfrak{n}), \operatorname{id}_{\mathfrak{g l}(V)}\right)$ consists of those $\mathfrak{n}$-modules $V$ that have a non-zero, $\mathfrak{n}$-trivial sub-quotient, provided $k \leqslant \operatorname{dim} \mathfrak{n}$, and otherwise is empty. However, both the ambient representation variety $\operatorname{Hom}_{\text {Lie }}(\mathfrak{n}, \mathfrak{g l}(V))$, and, a fortiori, the locus of representations having non-zero, $\mathfrak{n}$-trivial sub-quotients, may be difficult to compute explicitly. This is the reason why we chose to state Theorem 4.15 only for the Lie algebras $\mathfrak{g}=\mathfrak{s l}_{2}$ and $\mathfrak{g}=\mathfrak{s o l}_{2}$, where the explicit description from Lemma 4.14 is available.

\section{Algebras with linear Resonance}

In this section we continue our analysis of $\mathfrak{g}$-resonance varieties, focussing our attention on cdga's with linear resonance and zero differential. The guiding examples for this study are the cohomology rings of 1 -formal groups, such as the Artin groups.

Let $A^{\bullet}$ be a connected cdga, and let

$$
\mathscr{R}_{1}^{1}(A)=\left\{\alpha \in Z^{1}(A) \mid \exists \beta \in A^{1} \backslash \mathbb{C} \cdot \alpha \text { such that } d \beta+\alpha \beta=0 \text { in } A^{2}\right\}
$$

be its first resonance variety. Clearly, $\mathscr{R}_{1}^{1}(A)$ depends only on the differential $d: A^{1} \rightarrow A^{2}$ and on the multiplication map $\cup: A^{1} \wedge A^{1} \rightarrow A^{2}$; in fact, as noted in Lemma 4.1, the set $\mathscr{R}_{1}^{1}(A)$ depends only on the co-restriction of these maps to the subspace $\operatorname{im}(d)+\operatorname{im}(\cup) \subseteq A^{2}$.

Let $A^{\leqslant 2}=A / A^{>2}$ be the degree-2 truncation of $A$; by the above remarks, $\mathscr{R}_{1}^{1}(A)=\mathscr{R}_{1}^{1}\left(A^{\leqslant 2}\right)$. Note that a connected cdga $A \cdot$ that is concentrated in degrees at most 2 is encoded by a pair of linear maps, $d: A^{1} \rightarrow A^{2}$ and $\cup: A^{1} \wedge A^{1} \rightarrow A^{2}$. Moreover, cdga maps in this subcategory have an obvious linear algebra translation.

Definition 5.1. A connected cdga $A^{\bullet}$ has linear resonance if $\mathscr{R}_{1}^{1}(A)$ is a finite union of the form

$$
\mathscr{R}_{1}^{1}(A)=\bigcup_{C \in \mathscr{C}} C,
$$

where each $C$ is a linear subspace of $H^{1}(A)=Z^{1}(A)$.

As shown in [7], if $\pi$ is a finitely-generated, 1-formal group, and $A^{\bullet}=\left(H^{\bullet}(\pi, \mathbb{C}), d=0\right)$, then $A^{\bullet}$ has linear resonance. Moreover, as shown in [6], Gysin models of quasi-projective manifolds also have this property.

Given a connected cdga $A^{\bullet}$ and a linear subspace $C \subseteq A^{1}$, let $A_{\dot{C}}$ be the connected cdga concentrated in degrees $\leqslant 2$, defined by the restriction maps, $d_{C}: C \rightarrow A^{2}$ and $\cup_{C}: C \wedge C \rightarrow A^{2}$. Clearly, $A_{C}^{\cdot} \hookrightarrow A^{\leqslant 2}$ is a sub-cdga of the truncated cdga $A^{\leqslant 2}$. 
Lemma 5.2. Let $A^{\bullet}$ be a connected cdga with linear resonance, with $\mathscr{R}_{1}^{1}(A)$ decomposed as in (33). Then, for any Lie algebra $\mathfrak{g}$,

$$
\mathscr{F}(A, \mathfrak{g}) \supseteq \mathscr{F}^{1}(A, \mathfrak{g}) \cup \bigcup_{C \in \mathscr{C}} \mathscr{F}\left(A_{C}, \mathfrak{g}\right) .
$$

Moreover, if both $A^{1}$ and $\mathfrak{g}$ are finite-dimensional, then both $\mathscr{F}^{1}(A, \mathfrak{g})$ and $\mathscr{F}\left(A_{C}, \mathfrak{g}\right)$ are Zariskiclosed subsets of $\mathscr{F}(A, \mathfrak{g})$.

Proof. The first assertion follows from the naturality properties of the parameter set for flat connections. The second assertion follows from the equality $\mathscr{F}\left(A_{C}, \mathfrak{g}\right)=\mathscr{F}(A, \mathfrak{g}) \cap C \otimes \mathfrak{g}$.

Proposition 5.3. Suppose $A^{\bullet}$ is a cdga with zero differential and linear resonance. Then, if $\mathfrak{g}=\mathfrak{s l}_{2}$ or $\mathfrak{s o l}_{2}$, inclusion (34) becomes an equality.

Proof. An element $\omega \in A^{1} \otimes \mathfrak{g}$ may be written as $\omega=\alpha \otimes h+\beta \otimes x$ when $\mathfrak{g}=\mathfrak{s o l}_{2}$, and $\omega=\alpha \otimes H+\beta \otimes X+\gamma \otimes Y$ when $\mathfrak{g}=\mathfrak{s l}_{2}$. Let $U$ be the linear subspace of $A^{1}$ generated by $\alpha, \beta$ in the first case, and by $\alpha, \beta, \gamma$ in the second case. In either case, an easy computation shows that $\omega$ satisfies the flatness condition (10) if and only if $U$ is an isotropic subspace with respect to $\cup: A^{1} \wedge A^{1} \rightarrow A^{2}$.

Suppose $\omega \notin \mathscr{F}^{1}(A, \mathfrak{g})$, that is, $\operatorname{dim} U \geqslant 2$. Then clearly $U \subseteq \mathscr{R}_{1}^{1}(A)$. Now, by assumption, each irreducible component of $\mathscr{R}_{1}^{1}(A)$ is a linear subspace of $H^{1}(A)=A^{1}$. Hence, $U$ is contained in one of those linear subspaces, say, $U \subseteq C$. Therefore, $\omega \in \mathscr{F}(A, \mathfrak{g}) \cap C \otimes \mathfrak{g}=\mathscr{F}\left(A_{C}, \mathfrak{g}\right)$, and this completes the proof.

Theorem 5.4. Let $A^{\bullet}$ be a cdga with zero differential and linear resonance, and assume that $A^{1} \neq 0$. Let $\mathfrak{g}=\mathfrak{s l}_{2}$ or $\mathfrak{s o l}_{2}$, and let $\theta: \mathfrak{g} \rightarrow \mathfrak{g l}(V)$ be a finite-dimensional representation. If $\mathscr{R}_{1}^{1}(A)$ decomposes as in (33), then

$$
\mathscr{R}_{1}^{1}(A, \theta)=\Pi(A, \theta) \cup \bigcup_{C \in \mathscr{C}} \mathscr{F}\left(A_{C}, \mathfrak{g}\right) .
$$

Proof. First we prove that inclusion $\supseteq$ holds. In view of Corollary 3.8, we only need to show that $\mathscr{F}\left(A_{C}, \mathfrak{g}\right) \subseteq \mathscr{R}_{1}^{1}(A, \theta)$, for all $C \in \mathscr{C}$. To that end, let $\omega \in C \otimes \mathfrak{g}$ be an arbitrary element satisfying the Maurer-Cartan equation (10). The argument from the proof of Proposition 5.3 implies that $\omega \in U \otimes \mathfrak{g}$, where $U \subseteq A^{1}$ is an isotropic subspace. Using formula (13), we see that $d_{\omega}(U \otimes V)=0$. If $\omega$ is regular, i.e., $\operatorname{dim} U \geqslant 2$, then clearly $\omega \in \mathscr{R}_{1}^{1}(A, \theta)$, since $\operatorname{dim} d_{\omega}\left(A^{0} \otimes V\right) \leqslant \operatorname{dim} V$. Otherwise, $\omega$ must be of the form $\eta \otimes g$, for some $\eta \in C$. Now, by assumption, $C \subseteq \mathscr{R}_{1}^{1}(A)$. Hence, by Corollary 3.8, we again conclude that $\omega \in \mathscr{R}_{1}^{1}(A, \theta)$.

Next, we prove that inclusion $\subseteq$ holds. To that end, let $\omega \in \mathscr{R}_{1}^{1}(A, \theta)$, and suppose $\omega \notin$ $\bigcup_{C \in \mathscr{C}} \mathscr{F}\left(A_{C}, \mathfrak{g}\right)$. Then, by Proposition 5.3, $\omega$ is of the form $\eta \otimes g$. By Corollary 3.8, there is an eigenvalue $\lambda$ of $\theta(g)$ such that $\lambda \eta \in \mathscr{R}_{1}^{1}(A)$. If $\lambda \neq 0$, our linearity of resonance assumption implies that $\eta \in C$ for some $C \in \mathscr{C}$, a contradiction. Hence, $\omega \in \Pi(A, \theta)$, and we are done.

In the important case of 1-formal groups, Proposition 5.3 and Theorem 5.4 imply that the decomposition into irreducible components of the usual rank 1 resonance varieties in degree and depth 1 (guaranteed by [7]) determines the structure of both $\mathfrak{g}$-valued flat connections and higher-rank resonance varieties, in the same degree and depth, provided $\mathfrak{g}=\mathfrak{s l}_{2} \mathrm{Or}_{\mathfrak{s o l}}$. We will illustrate this phenomenon in the next two sections. 


\section{ARtin GROUPS}

We now turn our attention to a class of groups which plays a prominent role in geometric group theory and low-dimensional topology. For most of the section we will work with the restricted class of right-angled Artin groups, which are the most amenable to explicit computations in our setting, and then we will return towards the end to the wider class of Artin groups.

6.1. Rank 1 resonance. Let $\Gamma=(\mathrm{V}, \mathrm{E}, \ell)$ be a labeled, finite simplicial graph, with vertex set $\mathrm{V}$, edge set $\mathrm{E} \subseteq\left(\begin{array}{l}\mathrm{V} \\ 2\end{array}\right)$ and labeling function $\ell: \mathrm{E} \rightarrow \mathbb{Z}_{\geqslant 2}$. The Artin group associated to such a graph is the group $\pi_{\Gamma, \ell}$, with a generator $v$ for each vertex $v \in \mathrm{V}$, and a defining relation of the form $v w v \cdots=w v w \cdots$ for each edge $e=\{v, w\}$ in $\mathrm{E}$, where the words on each side of the equality are of length $\ell(e)$. As shown in [13], Artin groups are 1-formal.

An (unlabeled) finite simplicial graph $\Gamma=(\mathrm{V}, \mathrm{E})$ may be viewed as a labeled graph by setting $\ell(e)=2$, for each $e \in \mathrm{E}$. The corresponding group is called the right-angled Artin group (for short, raag) associated to $\Gamma$, and is simply denoted by $\pi_{\Gamma}$.

As is well-known, the cohomology algebra $A_{\Gamma}^{\cdot}=H^{\bullet}\left(\pi_{\Gamma}, \mathbb{C}\right)$ is isomorphic to the exterior Stanley-Reisner ring of the graph $\Gamma$. That is to say, $A_{\Gamma}$ is the quotient of the exterior $\mathbb{C}$-algebra on generators $v^{*}$, for all $v \in \mathrm{V}$, by the ideal generated by the monomials $v^{*} w^{*}$, for all $\{v, w\} \notin \mathrm{E}$. Let us endow this algebra with the differential $d=0$. The corresponding holonomy Lie algebra, $\mathfrak{h}(\Gamma):=\mathfrak{h}\left(A_{\Gamma}\right)$, has presentation

$$
\mathfrak{h}(\Gamma)=\mathbf{L}(\mathrm{V}) /([v, w]=0 \text { if }\{v, w\} \in \mathbf{E}) .
$$

Since the group $\pi_{\Gamma}$ is 1-formal, we know that the cohomology algebra $A_{\Gamma}^{\bullet}$ is a cdga with linear resonance, that is, the resonance variety $\mathscr{R}_{1}^{1}\left(A_{\Gamma}^{\bullet}\right)$ decomposes as a finite union of linear subspaces of $A_{\Gamma}^{1}=\mathbb{C}^{\mathrm{V}}$. This decomposition was worked out explicitly in [17, Theorem 5.5], as follows:

$$
\mathscr{R}_{1}^{1}\left(A_{\Gamma}^{\cdot}\right)=\bigcup_{\substack{\mathrm{W} \subseteq \mathrm{V} \\ c(\mathrm{~W})>1}} \mathbb{C}^{\mathrm{W}},
$$

where $c(\mathrm{~W})$ denotes the number of connected components of the induced subgraph $\Gamma_{\mathrm{W}}$, and $\mathbb{C}^{\mathrm{W}}$ denotes the coordinate subspace of $\mathbb{C}^{\mathrm{V}}$ spanned by $\mathrm{W}$.

6.2. Flat connections. We now analyze the space of flat $\mathfrak{g}$-connections on the algebra $A=A_{\Gamma} \cdot$ endowed with the zero differential, with the goal of making explicit the decomposition from Proposition 5.3.

Given a Lie algebra $\mathfrak{g}$, we will use the identification $\mathscr{F}(A, \mathfrak{g}) \cong \operatorname{Hom}_{\text {Lie }}(\mathfrak{h}(\Gamma), \mathfrak{g})$ induced by the natural isomorphism $\mathbb{C}^{\mathfrak{V}} \otimes \mathfrak{g} \cong \operatorname{Hom}(\mathbb{C}\langle\mathrm{V}\rangle, \mathfrak{g})$, as in Proposition 4.5 , and we will write elements $\omega \in \mathbb{C}^{\mathbb{V}} \otimes \mathfrak{g}$ as tuples of elements $\omega_{v} \in \mathfrak{g}$, indexed by the vertices $v \in \mathrm{V}$. In view of formula (36), $\omega$ belongs to $\mathscr{F}(A, \mathfrak{g})$ if and only if $\left[\omega_{u}, \omega_{v}\right]=0$ whenever $\{u, v\} \in \mathrm{E}$.

For each subset $\mathrm{W} \subseteq \mathrm{V}$, let $\mathrm{W}_{1}, \ldots, \mathrm{W}_{c}$ be the connected components of the vertex set of $\Gamma_{\mathrm{W}}$, let $\overline{\mathrm{W}}=\mathrm{V} \backslash \mathrm{W}$, and put

$$
S_{\mathrm{W}}=\left\{\omega \in \mathbb{C}^{\mathrm{V}} \otimes \mathfrak{g} \mid \begin{array}{ll}
\omega_{v}=0 & \text { for } v \in \overline{\mathrm{W}} \\
\operatorname{rank}\left\{\omega_{v}\right\}_{v \in \mathrm{W}_{i}} \leqslant 1 & \text { for } 1 \leqslant i \leqslant c
\end{array}\right\} .
$$

If $\mathfrak{g}$ is a finite-dimensional Lie algebra, then clearly $S_{\mathrm{W}}$ is a Zariski-closed subset of the affine space $\mathbb{C}^{\mathrm{W}} \otimes \mathfrak{g} \subseteq \mathbb{C}^{\mathrm{V}} \otimes \mathfrak{g}$. More specifically,

$$
S_{\mathrm{W}} \cong \prod_{i=1}^{c} \operatorname{cone}\left(\mathbb{P}\left(\mathbb{C}^{\mathrm{W}_{i}}\right) \times \mathbb{P}(\mathfrak{g})\right)
$$


Lemma 6.1. For every Lie algebra $\mathfrak{g}$, and every subset $\mathrm{W} \subseteq \mathrm{V}$, the following hold.

(1) If $c(\mathrm{~W})=1$, then $S_{\mathrm{W}} \subseteq \mathscr{F}^{1}\left(A_{\mathbb{C}^{\mathrm{W}}}, \mathfrak{g}\right)$.

(2) If $c(\mathrm{~W})>1$, then $S_{\mathrm{W}} \subseteq \mathscr{F}\left(A_{\mathbb{C} \mathrm{W}}, \mathfrak{g}\right)$.

Proof. Part (1) is immediate from the definitions.

To prove part (2), let $\omega \in S_{\mathrm{W}}$. Let $\{u, v\}$ be an edge in $\mathrm{E}$. If $\{u, v\} \nsubseteq \mathrm{W}$, then either $\omega_{u}=0$ or $\omega_{v}=0$, and so $\left[\omega_{u}, \omega_{v}\right]=0$. Otherwise, $\{u, v\} \subseteq \mathrm{W}_{i}$ for some $1 \leqslant i \leqslant c$; $\operatorname{thus,~} \operatorname{rank}\left\{\omega_{u}, \omega_{v}\right\} \leqslant 1$, and so again $\left[\omega_{u}, \omega_{v}\right]=0$. Therefore, $\omega \in \operatorname{Hom}_{\text {Lie }}(\mathfrak{h}(\Gamma), \mathfrak{g})$, and the desired conclusion follows.

Proposition 6.2. Let $\Gamma=(\mathrm{V}, \mathrm{E})$ be a finite simplicial graph, and let $\mathfrak{g}$ be a Lie algebra. Then $\mathscr{F}\left(A_{\Gamma}, \mathfrak{g}\right) \supseteq \bigcup_{\mathrm{W} \subseteq \mathrm{V}} S_{\mathrm{W}}$. Moreover, if $\mathfrak{g}=\mathfrak{s l}_{2}$ or $\mathfrak{s o l}_{2}$, then this inclusion holds as equality,

$$
\mathscr{F}\left(A_{\Gamma}, \mathfrak{g}\right)=\bigcup_{\mathrm{W} \subseteq \mathrm{V}} S_{\mathrm{W}} .
$$

Proof. The first claim follows at once from Lemmas 5.2 and 6.1 .

To prove the second claim, let $\omega \in \mathbb{C}^{\mathrm{V}} \otimes \mathfrak{g}$ be an arbitrary element, let $\operatorname{supp}(\omega)=\left\{v \in \mathrm{V} \mid \omega_{v} \neq\right.$ $0\}$ be its support, and let $\mathrm{V}=\mathrm{W}_{1} \amalg \cdots \amalg \mathrm{W}_{c} \amalg \overline{\mathrm{W}}$ be the partition associated to $\mathrm{W}=\operatorname{supp}(\omega)$. By construction, $\omega_{v}=0$ for all $v \in \overline{\mathrm{W}}$. For each $1 \leqslant i \leqslant c$ and each $u, v \in \mathrm{W}_{i}$, consider an edge-path in $\Gamma_{\mathrm{W}_{i}}$ connecting $u$ to $v$. If $\omega \in \operatorname{Hom}_{\text {Lie }}(\mathfrak{h}(\Gamma), \mathfrak{g})$, it follows from Remark 4.11 that $\operatorname{rank}\left\{\omega_{u}, \omega_{v}\right\} \leqslant 1$. Hence, $\omega \in S_{\mathrm{W}}$.

6.3. Higher-rank resonance. Let $\theta: \mathfrak{g} \rightarrow \mathfrak{g l}(V)$ be a finite-dimensional representation of a finite-dimensional Lie algebra $\mathfrak{g}$. We now describe a similar formula for the higher-rank resonance varieties $\mathscr{R}_{1}^{1}\left(A_{\Gamma}^{\cdot}, \theta\right)$, which makes explicit the decomposition from Theorem 5.4.

Given a subset $\mathrm{W} \subseteq \mathrm{V}$, put

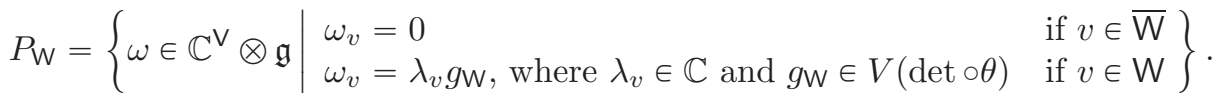

Clearly, $P_{\mathrm{W}}$ is a Zariski-closed subset of $\mathbb{C}^{\mathbb{V}} \otimes \mathfrak{g}$. Since the element $g_{\mathrm{W}} \in V(\operatorname{det} \circ \theta)$ is independent of the vertex $v \in \mathrm{W}$, we have that $\operatorname{rank}\left\{\omega_{v}\right\}_{v \in \mathrm{W}} \leqslant 1$, for every $\omega \in P_{\mathrm{W}}$. Thus, $P_{\mathrm{W}} \subseteq S_{\mathrm{W}}$.

Theorem 6.3. Let $\mathfrak{g}=\mathfrak{s l}_{2}$ or $\mathfrak{s o l}_{2}$. If $\Gamma=(\mathrm{V}, \mathrm{E})$ is a finite, simplicial graph, and $V_{\theta}$ is a finite-dimensional $\mathfrak{g}$-module, then

$$
\mathscr{R}_{1}^{1}\left(A_{\Gamma}^{\dot{\Gamma}}, \theta\right)=\bigcup_{\substack{\mathrm{W} \subseteq \mathrm{V} \\ c(\mathrm{~W})=1}} P_{\mathrm{W}} \cup \bigcup_{\substack{\mathrm{W} \subseteq \mathrm{V} \\ c(\mathrm{~W})>1}} S_{\mathrm{W}} .
$$

Proof. Let $A=A_{\Gamma}^{\cdot}$, equipped as before with the zero differential. In view of Theorem 5.4, the right-hand side of (42) is included in $\mathscr{R}_{1}^{1}(A, \theta)$. Indeed, we have that $P_{\mathrm{W}} \subseteq \Pi(A, \theta)$, for any subset $\mathrm{W} \subseteq \mathrm{V}$, by construction. Moreover, $S_{\mathrm{W}} \subseteq \mathscr{F}\left(A_{\mathbb{C}^{\mathrm{w}}}, \mathfrak{g}\right)$, for any subset $\mathrm{W} \subseteq \mathrm{V}$ with $c(\mathrm{~W})>1$, by Lemma 6.1.

To prove the reverse inclusion, let $\omega$ be a nonzero element in $\mathscr{R}_{1}^{1}(A, \theta) \subseteq \mathscr{F}(A, \mathfrak{g})$. By Proposition 6.2 , we have that $\omega \in S \mathrm{~W}$, for some $\mathrm{W} \subseteq \mathrm{V}$. If $\omega$ is regular, then necessarily $c(\mathrm{~W})>1$, since otherwise $S_{\mathrm{W}} \subseteq \mathscr{F}^{1}(A, \mathfrak{g})$, by Lemma 6.1. Thus, in order to finish the proof, we must show the following:

$$
\omega \in \mathscr{F}^{1}(A, \mathfrak{g}) \backslash \bigcup_{c(\mathrm{~W})>1} S_{\mathrm{W}} \Rightarrow \omega \in \bigcup_{c(\mathrm{~W})=1} P_{\mathrm{W}} .
$$


Set $\mathrm{W}=\operatorname{supp}(\omega)$. As we saw in the proof of Proposition $6.2, \omega \in S_{\mathrm{W}}$, and so the induced subgraph $\Gamma_{\mathrm{W}}$ is connected. Theorem 5.4 tells us that either $\omega \in \Pi(A, \theta)$, or $\omega \in P\left(\mathbb{C}^{\mathrm{W}^{\prime}} \times \mathfrak{g}\right)$, for some subset $\mathrm{W}^{\prime} \subseteq \mathrm{V}$ with $c\left(\mathrm{~W}^{\prime}\right)>1$. In the first case, it follows that $\omega \in P_{\mathrm{W}}$, and we are done. In the second case, we must have $\mathrm{W} \subseteq \mathrm{W}^{\prime}$, and so $\mathrm{W} \subseteq \mathrm{W}_{i}^{\prime}$, for some $1 \leqslant i \leqslant c\left(\Gamma_{\mathrm{W}^{\prime}}\right)$. Since $\omega \in \mathscr{F}^{1}(A, \mathfrak{g})$ and $\mathrm{W}=\operatorname{supp}(\omega)$, this implies $\omega \in S_{\mathrm{W}^{\prime}}$, a contradiction.

6.4. Irreducible components. In the case when $\mathfrak{g}=\mathfrak{s l}_{2}$, we may use Proposition 6.2 and Theorem 6.3 to find the decompositions of $\mathscr{F}\left(A_{\Gamma}^{\bullet}, \mathfrak{g}\right)$ and $\mathscr{R}_{1}^{1}\left(A_{\Gamma}^{\bullet}, \theta\right)$ into irreducible components.

To that end, we need to introduce a partial order among the subsets of the vertex set $\mathrm{V}$. For each inclusion $\mathrm{W} \subseteq \mathrm{W}^{\prime}$ of vertex subsets, let $K_{\mathrm{WW}^{\prime}}:\left\{\mathrm{W}_{1}, \ldots, \mathrm{W}_{c}\right\} \rightarrow\left\{\mathrm{W}_{1}^{\prime}, \ldots, \mathrm{W}_{c^{\prime}}^{\prime}\right\}$ be the induced map from the connected components of $\Gamma_{\mathrm{W}}$ to the connected components of $\Gamma_{\mathrm{W}^{\prime}}$. The order relation on the subsets of $\mathrm{V}$ is then given by

$$
\mathrm{W} \leqslant \mathrm{W}^{\prime} \Leftrightarrow \mathrm{W} \subseteq \mathrm{W}^{\prime} \text { and } K_{\mathrm{WW}^{\prime}} \text { is injective. }
$$

Clearly, if $c(\mathbf{W})>1$ and $c\left(\mathbf{W}^{\prime}\right)=1$, then $\mathbf{W} \leqslant \mathbf{W}^{\prime}$. Furthermore, if $c(\mathbf{W})=1$, then $\mathbf{W} \leqslant \mathbf{W}^{\prime}$ if and only if $\mathrm{W} \subseteq \mathrm{W}^{\prime}$.

Lemma 6.4. With notation as above, the following hold.

(1) $S_{\mathrm{W}} \subseteq S_{\mathrm{W}^{\prime}} \Leftrightarrow \mathrm{W} \leqslant \mathrm{W}^{\prime}$, for all $\mathrm{W}, \mathrm{W}^{\prime}$.

(2) $S_{\mathrm{W}} \nsubseteq P_{\mathrm{W}^{\prime}}$, provided $c(\mathrm{~W})>1$ and $c\left(\mathrm{~W}^{\prime}\right)=1$.

(3) $P_{\mathrm{W}} \subseteq P_{\mathrm{W}^{\prime}} \Leftrightarrow \mathrm{W} \leqslant \mathrm{W}^{\prime}$, provided $c(\mathrm{~W})=c\left(\mathrm{~W}^{\prime}\right)=1$.

(4) $P_{\mathrm{W}} \subseteq S_{\mathrm{W}^{\prime}} \Leftrightarrow \mathrm{W} \leqslant \mathrm{W}^{\prime}$, provided $c(\mathrm{~W})=1$ and $c\left(\mathrm{~W}^{\prime}\right)>1$.

Proof. We start with part (1). The implication $\mathrm{W} \leqslant \mathrm{W}^{\prime} \Rightarrow S_{\mathrm{W}} \subseteq S_{\mathrm{W}^{\prime}}$ is immediate. Conversely, suppose $S_{\mathrm{W}} \subseteq S_{\mathrm{W}^{\prime}}$. Then clearly $\mathrm{W} \subseteq \mathrm{W}^{\prime}$, by a support argument. It remains to show that $K_{\mathrm{WW}^{\prime}}$ is injective. If that were not the case, there would be two distinct connected components of $\Gamma_{\mathrm{W}}$ such that their vertex sets, call them $\mathrm{W}_{1}$ and $\mathrm{W}_{2}$, are both included in a component $\mathrm{W}_{1}^{\prime}$ of $\Gamma_{\mathrm{W}^{\prime}}$. Now pick $v_{i} \in \mathrm{W}_{i}$ and $g_{i} \in \mathfrak{g}$ such that $\operatorname{rank}\left\{g_{1}, g_{2}\right\}=2$, and define an element $\omega \in \mathbb{C}^{\mathbb{V}} \otimes \mathfrak{g}$ by setting $\omega_{v_{i}}=g_{i}$ and $\omega_{v}=0$, otherwise. We then have $\omega \in S_{\mathrm{W}} \backslash S_{\mathrm{W}^{\prime}}$, a contradiction.

To prove part (2), suppose that $S_{\mathrm{W}} \subseteq P_{\mathrm{W}^{\prime}}$. Then, since $P_{\mathrm{W}^{\prime}} \subseteq S_{\mathrm{W}^{\prime}}$, we must have $S_{\mathrm{W}} \subseteq S_{\mathrm{W}^{\prime}}$. By part (1), this implies $\mathrm{W} \leqslant \mathrm{W}^{\prime}$, and so $K_{\mathrm{WW}^{\prime}}$ must be injective, contradicting the assumption that $c(\mathrm{~W})>c\left(\mathrm{~W}^{\prime}\right)$.

Before proceeding with the last two parts, recall from Lemma 3.9 that there are two possibilities for the variety $V(\operatorname{det} \circ \theta)$ : it is either equal to $\mathfrak{g}$, or to $V$ (det). In the first case, we have that $P_{\mathrm{W}}=S_{\mathrm{W}}$, for all $\mathrm{W} \subseteq \mathrm{V}$ with $c(\mathrm{~W})=1$, and so parts (3) and (4) follow at once from part (1). In the second case, let us pick a non-zero matrix $g \in V$ (det), and prove parts (3) and (4) separately.

For part (3), suppose that $\mathbf{W} \subseteq \mathbf{W}^{\prime}$. Since we are assuming that $c(\mathbf{W})=c\left(\mathbf{W}^{\prime}\right)=1$, we must have $P_{\mathrm{W}} \subseteq P_{\mathrm{W}^{\prime}}$, by part (1). Conversely, suppose $\mathrm{W} \nsubseteq \mathrm{W}^{\prime}$. Then pick $v \in \mathrm{W} \backslash \mathrm{W}^{\prime}$, and define an element $\omega \in \mathbb{C}^{\mathrm{V}} \otimes \mathfrak{g}$ by setting $\omega_{v}=g$ and $\omega_{u}=0$ for $u \neq v$. Clearly, $\omega \in P_{\mathrm{W}} \backslash P_{\mathrm{W}^{\prime}}$, and so $P_{\mathrm{W}} \nsubseteq P_{\mathrm{W}^{\prime}}$.

For part (4), suppose that $\mathrm{W} \subseteq \mathrm{W}^{\prime}$. Then, since $c(\mathrm{~W})=1$, we must have $P_{\mathrm{W}} \subseteq S_{\mathrm{W}^{\prime}}$, again by part (1). Conversely, suppose $v \in \mathbf{W} \backslash \mathbf{W}^{\prime}$. If we set $\omega_{v}=g$ and $\omega_{u}=0$ for $u \neq v$, we then have $\omega \in P_{\mathrm{W}} \backslash S_{\mathrm{W}^{\prime}}$, and this finishes the proof. 
Corollary 6.5. Let $\Gamma$ be a finite, simplicial graph, and let $\theta: \mathfrak{s l}_{2} \rightarrow \mathfrak{g l}(V)$ be a finite-dimensional representation. We then have the following decompositions into irreducible components:

$$
\begin{aligned}
& \mathscr{F}\left(A_{\Gamma}^{\cdot}, \mathfrak{s l}_{2}\right)=\bigcup_{\mathrm{W} \leqslant- \text { maximal }} S_{\mathrm{W}}, \\
& \mathscr{R}_{1}^{1}\left(A_{\Gamma}^{\dot{0}}, \theta\right)=\bigcup_{\substack{c(\mathrm{~W})=1 \\
\mathrm{~W} \leqslant- \text { maximal }}} P_{\mathrm{W}} \cup \underset{\substack{c(\mathrm{~W})>1 \\
\nexists \mathrm{W} \supsetneqq \mathrm{W}^{\prime} \text { with } c\left(\mathrm{~W}^{\prime}\right)>1}}{ } S \text {. }
\end{aligned}
$$

Proof. For any subset $\mathrm{W}$ of the vertex set $\mathrm{V}$, both $P_{\mathrm{W}}$ and $S_{\mathrm{W}}$ are irreducible, Zariski-closed subsets of $\mathscr{F}\left(A_{\Gamma}^{\cdot}, \mathfrak{s l}_{2}\right)$. Indeed, the first set equals $P\left(\mathbb{C}^{\mathrm{W}} \times V(\operatorname{det} \circ \theta)\right)$, which is irreducible by Lemma 3.9, while the second set equals $\prod_{i=1}^{c(\mathrm{~W})} P\left(\mathbb{C}^{\mathrm{W}_{i}} \times \mathfrak{s l}_{2}\right)$, which is clearly irreducible. Using Lemma 6.4 to eliminate redundancies in (40) and (42) completes the proof.

6.5. Semisimple Lie algebras. Our emphasis on the $\mathfrak{s l}_{2}$ case comes from the fact that the inclusion from Proposition 6.2 may be strict for arbitrary Lie algebras $\mathfrak{g}$ and graphs $\Gamma$. More precisely, we have the following result.

Proposition 6.6. Suppose $\mathfrak{g}$ is a semisimple Lie algebra, different from $\mathfrak{s l}_{2}$. There is then a connected, finite simple graph $\Gamma$ such that $\mathscr{F}\left(A_{\Gamma}^{\cdot}, \mathfrak{g}\right) \neq \bigcup_{\mathrm{W} \subseteq \mathrm{V}} S_{\mathrm{W}}$.

Proof. For each such $\mathfrak{g}$, we will construct a connected, finite simple graph $\Gamma$ and a regular element $\omega \in \mathscr{F}\left(A_{\Gamma}, \mathfrak{g}\right)$ with the property that $\omega_{v} \neq 0$ for all $v \in \mathrm{V}$. This will imply $\omega \notin \bigcup_{\mathrm{W} \subseteq \mathrm{V}} S_{\mathrm{W}}$. Indeed, if $\omega \in S_{\mathrm{W}}$, then necessarily $\mathrm{W}=\mathrm{V}$, since $\operatorname{supp}(\omega)=\mathrm{V}$; see Lemma 6.1. More precisely, it follows from Lemma 6.1(1) that $\omega \in \mathscr{F}^{1}\left(A_{\Gamma}^{\cdot}, \mathfrak{g}\right)$, contradicting regularity.

First, we need to recall some standard facts on semisimple Lie algebras, cf. [12]. Let $r$ be the dimension of a Cartan subalgebra of $\mathfrak{g}$; the assumption that $\mathfrak{g} \neq \mathfrak{s l}_{2}$ means that $r>1$. Pick a system of simple roots $\left\{\alpha_{1}, \ldots, \alpha_{r}\right\}$. For an arbitrary root $\alpha$, pick a generator $g_{\alpha}$ of the root space $\mathfrak{g}_{\alpha} \subseteq \mathfrak{g}$. Then the family $\left\{g_{\alpha}\right\}_{\alpha}$ is independent, $\left[g_{\alpha_{i}}, g_{-\alpha_{j}}\right]=0$ for $i \neq j$, and $\left[g_{\alpha}, g_{\beta}\right]=0$ if $0 \neq \alpha+\beta$ is not a root. Choose a positive root $\gamma$ of maximal height. Then $\left[g_{\gamma}, g_{\alpha_{i}}\right]=\left[g_{-\gamma}, g_{-\alpha_{i}}\right]=0$, for all $1 \leqslant i \leqslant r$.

If $r>2$, we let $\Gamma$ be the graph with vertex set $\mathrm{V}=\left\{ \pm \alpha_{1}, \ldots, \pm \alpha_{r}\right\}$ and edges $\left\{\alpha_{i},-\alpha_{j}\right\}$ for $i \neq j$. Clearly, $\Gamma$ is connected. Now set $\omega_{\alpha}=g_{\alpha}$ for $\alpha \in \mathrm{V}$. Then $\omega \in \mathscr{F}\left(A_{\Gamma}^{\dot{r}} \mathfrak{g}\right)$ is regular and $\operatorname{supp}(\omega)=\mathrm{V}$, as needed.

If $r=2$ and $\mathfrak{g} \neq \mathfrak{s l}_{2} \times \mathfrak{s l}_{2}$, we let $\mathrm{V}=\left\{ \pm \alpha_{1}, \pm \alpha_{2}, \pm \gamma\right\}$ and declare the edges to be $\left\{\alpha_{i},-\alpha_{j}\right\}$ for $i \neq j$ and $\left\{\epsilon \alpha_{i}, \epsilon \gamma\right\}$, with $\epsilon= \pm 1$, while if $\mathfrak{g}=\mathfrak{s l}_{2} \times \mathfrak{s l}_{2}$, we let $\mathrm{V}=\left\{ \pm \alpha_{1}, \pm \alpha_{2}\right\}$ and declare the edges to be $\left\{\alpha_{i},-\alpha_{j}\right\}$ for $i \neq j$ and $\left\{\epsilon \alpha_{1}, \epsilon \alpha_{2}\right\}$, with $\epsilon= \pm 1$. In both cases, the resulting graph $\Gamma$ is connected, and the desired form $\omega$ is constructed as before.

6.6. Labeled graphs and odd contractions. We now return to the general case of a (finitely generated) Artin group. A construction described in [7, \$11.9] associates to each labeled, finite simplicial graph $(\Gamma, \ell)$ an unlabeled graph, $\tilde{\Gamma}$, called the odd contraction of $(\Gamma, \ell)$, as follows. We first define an unlabeled graph $\Gamma_{\text {odd }}$ by keeping all the vertices of $\Gamma$, and retaining only those edges for which the label is odd (after which the label is discarded). We then let $\tilde{\Gamma}$ be the graph whose vertices correspond to the connected components of $\Gamma_{\text {odd }}$, with two distinct components determining an edge $\left\{c, c^{\prime}\right\}$ in $\tilde{\Gamma}$ if and only if there exist vertices $v \in c$ and $v^{\prime} \in c^{\prime}$ which are connected by an edge in $\Gamma$. 
Let $\pi_{\Gamma, \ell}$ be the Artin group associated to the labeled graph $(\Gamma, \ell)$, and let $\pi_{\tilde{\Gamma}}$ be the right-angled Artin group associated to the odd contraction of $(\Gamma, \ell)$. Furthermore, let $A_{\Gamma, \ell}^{\cdot}=H^{\bullet}\left(\pi_{\Gamma, \ell}, \mathbb{C}\right)$ and $A_{\tilde{\Gamma}}=H^{\bullet}\left(\pi_{\tilde{\Gamma}}, \mathbb{C}\right)$ be the respective cohomology algebras, both endowed with the zero differential.

Proposition 6.7. For each finite-dimensional Lie algebra $\mathfrak{g}$ and each finite-dimensional representation $\theta: \mathfrak{g} \rightarrow \mathfrak{g l}(V)$, there is an isomorphism

$$
\left(\mathscr{F}\left(A_{\Gamma, \ell}^{\bullet}, \mathfrak{g}\right), \mathscr{R}_{1}^{1}\left(A_{\dot{\Gamma}, \ell}^{\bullet}, \theta\right)\right) \cong\left(\mathscr{F}\left(A_{\tilde{\Gamma}}^{\dot{\Gamma}}, \mathfrak{g}\right), \mathscr{R}_{1}^{1}\left(A_{\tilde{\Gamma}}^{\dot{\Gamma}}, \theta\right)\right)
$$

between the corresponding pairs of affine varieties.

Proof. As shown in [7, Lemma 11.11], the groups $\pi_{\Gamma, \ell}$ and $\pi_{\tilde{\Gamma}}$ have the same Malcev-Lie algebra, and thus the same Sullivan 1-minimal model. In particular, the cdga's $A_{\dot{\Gamma}, \ell}$ and $A_{\tilde{\Gamma}}$ have the same co-restrictions in low degrees. Lemma 4.1, then, yields the desired isomorphism.

When combined with Corollary 6.5, this proposition produces explicit decompositions into irreducible components for the varieties $\mathscr{F}\left(A_{\Gamma, \ell}^{\cdot}, \mathfrak{s l}_{2}\right)$ and $\mathscr{R}_{1}^{1}\left(A_{\Gamma, \ell}^{\cdot}, \theta\right)$, for any labeled, finite simplicial graph $(\Gamma, \ell)$ and any finite-dimensional representation $\theta: \mathfrak{s l}_{2} \rightarrow \mathfrak{g l}(V)$.

6.7. Germs of representation varieties. To conclude this section, let us compare our computations with the Kapovich-Millson universality theorem [13]. This striking result (Theorem 1.9 from [13]) says the following: Given a point $x$ on an affine variety $\mathcal{X}$ defined over $\mathbb{Q}$, there is a labeled finite simplicial graph $(\Gamma, \ell)$ and a (nontrivial) representation $\rho: \pi_{\Gamma, \ell} \rightarrow \mathrm{PSL}_{2}$ with finite image and trivial centralizer such that the germ of the GIT quotient of the representation variety at the class of $\rho$ is isomorphic to the germ of the given singularity; that is,

$$
\left(\operatorname{Hom}\left(\pi_{\Gamma, \ell}, \mathrm{PSL}_{2}\right) / / \mathrm{PSL}_{2}\right)_{([\rho])} \cong \mathcal{X}_{(x)} .
$$

Moreover, Kapovich and Millson show in the proof of [13, Theorem 14.7] that the representation variety admits a local cross-section for the conjugation action of $\mathrm{PSL}_{2}$ at the point $\rho$. Therefore,

$$
\operatorname{Hom}\left(\pi_{\Gamma, \ell}, \mathrm{PSL}_{2}\right)_{(\rho)} \cong \mathcal{X}_{(x)} \times \mathbb{C}_{(0)}^{3}
$$

as analytic germs.

So what can be said at the trivial representation? By Theorem 17.3 from [13], the variety $\operatorname{Hom}\left(\pi_{\Gamma, \ell}, \mathrm{PSL}_{2}\right)$ has at worst a quadratic singularity at the origin $\rho=1$. Our work complements this remarkable result, as follows.

Let $\tilde{\Gamma}$ be the odd contraction of $(\Gamma, \ell)$. It follows from Theorem 2.9 and Proposition 6.7 that we have a local analytic isomorphism

$$
\operatorname{Hom}\left(\pi_{\Gamma, \ell}, \mathrm{PSL}_{2}\right)_{(1)} \cong \mathscr{F}\left(H^{\bullet}\left(\pi_{\tilde{\Gamma}}, \mathbb{C}\right), \mathfrak{s l}_{2}\right)_{(0)}
$$

which identifies $\mathscr{V}_{1}^{1}\left(K\left(\pi_{\Gamma, \ell}, 1\right), \iota\right)_{(1)}$ with $\mathscr{R}_{1}^{1}\left(H^{\bullet}\left(\pi_{\tilde{\Gamma}}, \mathbb{C}\right), \theta\right)_{(0)}$, for every rational representation $\iota: \mathrm{PSL}_{2} \rightarrow \mathrm{GL}(V)$. Proposition 6.2 then says that the analytic singularity at 1 of the representation variety $\operatorname{Hom}\left(\pi_{\Gamma, \ell}, \mathrm{PSL}_{2}\right)$ can be completely described in terms of the graph $\tilde{\Gamma}$. Moreover, according to Theorem 6.3 , the same thing happens with the nonabelian characteristic varieties $\mathscr{V}_{1}^{1}\left(K\left(\pi_{\Gamma, \ell}, 1\right), \iota\right)$, which can be completely described in terms of the graph $\tilde{\Gamma}$ and the tangential representation of $\iota$. 


\section{QUASI-PROJECTIVE MANIFOLDS}

We devote this last section to the $\mathfrak{g}$-resonance varieties associated to an irreducible, smooth, quasi-projective variety $X$. For the most part, we emphasize the case when $X$ is 1 -formal and $\mathfrak{g}=\mathfrak{s l}_{2}$, a case in which our methods lead to a rather explicit description of the variety of $\mathfrak{g}$-valued flat connections and of the higher-rank resonance varieties, in degree 1 and depth 1.

7.1. Admissible maps onto curves. Let $X$ be a quasi-projective manifold. Up to reparametrization at the target, the variety $X$ admits finitely many admissible maps $f: X \rightarrow S$ onto connected, smooth complex curves with $\chi(S)<0$. Admissibility means that $f$ is regular, and has connected generic fiber. As shown by Arapura in [1], the correspondence $f \mapsto f^{*}\left(\mathbb{T}\left(\pi_{1}(S)\right)\right)$ establishes a bijection between the set $\mathscr{E}_{X}$ of equivalence classes of admissible maps from $X$ to a curve $S$ with $\chi(S)<0$ and the set of positive-dimensional, irreducible components of $\mathscr{V}_{1}^{1}(X)$ containing 1.

Next, we recall from [6, Theorem $\mathrm{C}$ and Example 5.3] the relationship between $\mathscr{E}_{X}$ and the linear decomposition of $\mathscr{R}_{1}^{1}(A)$, for a convenient choice of Gysin model $A^{\bullet}$ of $X$. Each admissible map $f$ induces an injective cdga map $f^{*}: A^{\bullet}(S) \hookrightarrow A^{\bullet}$, where $A^{\bullet}(S)$ is the canonical Gysin model of the curve $S$ and has the property that $A^{>2}(S)=0$.

Due to the equivalences detailed in (15), we will assume from now on that $b_{1}(X)>0$. In this case, the (linear) irreducible decomposition of $\mathscr{R}_{1}^{1}(A)$ is given by

$$
\mathscr{R}_{1}^{1}(A)=\{0\} \cup \bigcup_{f \in \mathscr{E}_{X}} f^{*}\left(H^{1}(A(S))\right),
$$

with the convention that $\{0\}$ is omitted when $\mathscr{E}_{X} \neq \varnothing$.

7.2. Flat connections and resonance varieties. Now let $\mathfrak{g}$ be a Lie algebra. Given an admissible map $f: X \rightarrow S$, let us denote by $f^{!}$the linear map $f^{1} \otimes \mathrm{id}_{\mathfrak{g}}: A^{1}(S) \otimes \mathfrak{g} \rightarrow A^{1} \otimes$ $\mathfrak{g}$. Since the homomorphism $f^{*}: A^{\bullet}(S) \rightarrow A^{\bullet}$ is injective, the map $f^{!}$is also injective. By naturality of the parameter space for flat $\mathfrak{g}$-connections, the map $f^{!}$restricts to an injective map $f^{!}: \mathscr{F}\left(A^{\bullet}(S), \mathfrak{g}\right) \hookrightarrow \mathscr{F}\left(A^{\bullet}, \mathfrak{g}\right)$. Furthermore,

$$
f^{!}(\mathscr{F}(A(S), \mathfrak{g}))=\mathscr{F}(A, \mathfrak{g}) \cap f^{*}\left(A^{1}(S)\right) \otimes \mathfrak{g} .
$$

Let $\theta: \mathfrak{g} \rightarrow \mathfrak{g l}(V)$ be a finite-dimensional representation. We then have the following analog of Proposition 5.3 and Theorem 5.4.

Proposition 7.1. Let $X$ be a quasi-projective manifold, and let $A^{\bullet}$ be a convenient Gysin model for $X$, as above. Then, the following inclusions hold:

$$
\begin{gathered}
\mathscr{F}(A, \mathfrak{g}) \supseteq \mathscr{F}^{1}(A, \mathfrak{g}) \cup \bigcup_{f \in \mathscr{E}_{X}} f^{!}(\mathscr{F}(A(S), \mathfrak{g})), \\
\mathscr{R}_{1}^{1}(A, \theta) \supseteq \Pi(A, \theta) \cup \bigcup_{f \in \mathscr{E}_{X}} f^{!}(\mathscr{F}(A(S), \mathfrak{g})),
\end{gathered}
$$

where, in both instances, $f$ runs through the set of equivalence classes of admissible maps from $X$ to curves $S$ with $\chi(S)<0$. Moreover, if $\theta=\mathrm{id}_{\mathbb{C}}$, then both these inclusions become equalities.

Proof. Inclusion (52) is immediate, while inclusion (53) follows from Corollary 3.8 and Lemma 2.6(2), combined with Proposition 2.4.

Finally, assume that $\theta=\mathrm{id}_{\mathbb{C}}$. Equality in $(52)$ is then obvious, since $\mathscr{F}(A, \mathbb{C})=\mathscr{F}^{1}(A, \mathbb{C})$, while equality in (53) is equivalent to equality (50), which we know holds. 
7.3. The 1-formal situation. Now consider the case when $X$ is a 1-formal, quasi-projective manifold, for instance, a smooth, projective variety, or the complement of a hypersurface in a complex projective space. In this case, Gysin models may be efficiently replaced by cohomology rings endowed with the zero differential, as discussed in Example 2.11.

It follows from [7, Theorem C and Proposition 7.2(3)] that the cohomology algebra of a 1formal, quasi-projective manifold $X$ has linear resonance, in the sense of Definition 5.1. More precisely, the decomposition of $\mathscr{R}_{1}^{1}(X)$ into (linear) irreducible components is given by

$$
\mathscr{R}_{1}^{1}(X)=\{0\} \cup \bigcup_{f \in \mathscr{E}_{X}} f^{*}\left(H^{1}(S, \mathbb{C})\right),
$$

again with the convention that $\{0\}$ is omitted when $\mathscr{E}_{X} \neq \varnothing$. Moreover, the induced homomorphism, $f^{*}: H^{\bullet}(S, \mathbb{C}) \rightarrow H^{\bullet}(X, \mathbb{C})$, is an embedding of cohomology rings, and $H^{>2}(S, \mathbb{C})=0$.

Corollary 7.2. Let $X$ be a 1-formal, quasi-projective manifold with $b_{1}(X)>0$. Let $\mathfrak{g}=\mathfrak{s l}_{2}$ or $\mathfrak{s o l}_{2}$, and let $\theta: \mathfrak{g} \rightarrow \mathfrak{g l}(V)$ be a finite-dimensional representation. Then, the following equalities hold:

$$
\begin{aligned}
\mathscr{F}\left(H^{\bullet}(X, \mathbb{C}), \mathfrak{g}\right) & =\mathscr{F}^{1}\left(H^{\bullet}(X, \mathbb{C}), \mathfrak{g}\right) \cup \bigcup_{f \in \mathscr{E}_{X}} f^{!}\left(\mathscr{F}\left(H^{\bullet}(S, \mathbb{C}), \mathfrak{g}\right)\right), \\
\mathscr{R}_{1}^{1}\left(H^{\bullet}(X, \mathbb{C}), \theta\right) & =\Pi\left(H^{\bullet}(X, \mathbb{C}), \theta\right) \cup \bigcup_{f \in \mathscr{E}_{X}} f^{!}\left(\mathscr{F}\left(H^{\bullet}(S, \mathbb{C}), \mathfrak{g}\right)\right),
\end{aligned}
$$

where, in both instances, $f$ runs through the set of equivalence classes of admissible maps from $X$ to curves $S$ with $\chi(S)<0$.

Proof. For an admissible function $f: X \rightarrow S$, write $C=f^{*}\left(H^{1}(S, \mathbb{C})\right)$. As noted in the proof of Lemma 5.2, the set $\mathscr{F}\left(H^{\bullet}(X, \mathbb{C})_{C}, \mathfrak{g}\right)$ coincides with $\mathscr{F}\left(H^{\bullet}(X, \mathbb{C}), \mathfrak{g}\right) \cap C \otimes \mathfrak{g}$. In turn, the latter set coincides with $f^{!}\left(\mathscr{F}\left(H^{\bullet}(S, \mathbb{C}), \mathfrak{g}\right)\right)$, since $f^{*}: H^{\bullet}(S, \mathbb{C}) \rightarrow H^{\bullet}(X, \mathbb{C})$ is injective. The result now follows from Proposition 5.3 and Theorem 5.4, together with formula (54).

7.4. Flat connections on curves. Our next objective is to extract from formulas (55) and (56) the irreducible decompositions of the varieties $\mathscr{F}\left(H^{\bullet}(X, \mathbb{C}), \mathfrak{g}\right)$ and $\mathscr{R}_{1}^{1}\left(H^{\bullet}(X, \mathbb{C}), \theta\right)$. Before proceeding, let us first analyze in more detail the contribution made by each admissible map.

Lemma 7.3. Let $S$ be a curve with $\chi(S)<0$, and let $\mathfrak{g}=\mathfrak{s l}_{2}$ or $\mathfrak{s o l}_{2}$. Then $\mathscr{F}(H \cdot(S, \mathbb{C}), \mathfrak{g})$ is irreducible and contains regular elements.

Proof. First suppose $S$ is non-compact. Let $n=\operatorname{dim} H^{1}(S, \mathbb{C})$; since $\chi(S)<0$, we must have $n \geqslant$ 2. Since $H^{2}(S, \mathbb{C})=0$, the cup-product map on $H^{1}(S, \mathbb{C})$ is trivial. Hence, $\mathscr{F}\left(H^{\bullet}(S, \mathbb{C}), \mathfrak{g}\right)=\mathfrak{g}^{n}$, and the desired conclusions follow, since $1<\operatorname{dim} \mathfrak{g}$.

Next, suppose $S$ is compact. Let $g=\frac{1}{2} \operatorname{dim} H^{1}(S, \mathbb{C})$ be the genus of the curve; since $\chi(S)<0$, we must have $g \geqslant 2$. The cup-product map, $\cup: \bigwedge^{2} H^{1}(S, \mathbb{C}) \rightarrow H^{2}(S, \mathbb{C})=\mathbb{C}$, defines a symplectic inner product $\langle\cdot, \cdot\rangle$ on $H^{1}(S, \mathbb{C})$. Let us fix a symplectic basis $\left\{u_{1}, v_{1}, \ldots, u_{g}, v_{g}\right\}$, so that $\left\langle u_{i}, u_{j}\right\rangle=\left\langle v_{i}, v_{j}\right\rangle=0$ and $\left\langle u_{i}, v_{j}\right\rangle=\delta_{i j}$ for all $i, j$. Let $\mathrm{Sp}_{2 g}$ be the linear, connected automorphism group of $\langle\cdot, \cdot\rangle$, and let $U=\operatorname{span}\left\{u_{1}, \ldots, u_{g}\right\}$ be the associated maximal isotropic subspace.

Set $r=\operatorname{dim} \mathfrak{g}$. As we saw in the proof of Proposition 5.3 , the subvariety $\mathscr{F}\left(H^{\bullet}(S, \mathbb{C}), \mathfrak{g}\right) \subseteq$ $H^{1}(S, \mathbb{C}) \otimes \mathfrak{g}$ may be identified with the set of $r$-tuples $\left(\alpha_{1}, \ldots, \alpha_{r}\right) \in\left(H^{1}(S, \mathbb{C})\right)^{r}$ spanning an 
isotropic subspace with respect to $\cup$. Consider the regular map

$$
\Phi: \operatorname{Sp}_{2 g} \times U^{r} \rightarrow \mathscr{F}\left(H^{\bullet}(S, \mathbb{C}), \mathfrak{g}\right), \quad\left(s, \beta_{1}, \ldots, \beta_{r}\right) \mapsto\left(s \beta_{1}, \ldots, s \beta_{r}\right) .
$$

As is well-known (see for instance [15, Ch. I]), every maximal isotropic subspace of $H^{1}(S, \mathbb{C})$ is of the form $s(U)$, for some $s \in \mathrm{Sp}_{2 g}$. Thus, the map $\Phi$ is surjective, and the desired conclusions follow.

Note that our proof actually gives an explicit description of the subvariety $f^{!}\left(\mathscr{F}\left(H^{\bullet}(S, \mathbb{C}), \mathfrak{g}\right)\right) \subseteq$ $\mathscr{F}\left(H^{\bullet}(X, \mathbb{C}), \mathfrak{g}\right)$.

7.5. Irreducible decomposition. We are now in a position to state and prove the main results of this section.

Theorem 7.4. Let $X$ be a 1-formal, quasi-projective manifold with $b_{1}(X)>0$, and let $\mathfrak{g}=\mathfrak{s l}_{2}$ or $\mathfrak{s o l}_{2}$. Then, the irreducible decomposition of $\mathscr{F}\left(H^{\bullet}(X, \mathbb{C}), \mathfrak{g}\right)$ is given by

$$
\mathscr{F}\left(H^{\bullet}(X, \mathbb{C}), \mathfrak{g}\right)= \begin{cases}P\left(H^{1}(X, \mathbb{C}) \times \mathfrak{g}\right) & \text { if } \mathscr{R}_{1}^{1}(X)=\{0\}, \\ f^{!}(\mathscr{F}(H \bullet(S, \mathbb{C}), \mathfrak{g})) & \text { if } \mathscr{R}_{1}^{1}(X)=H^{1}(X, \mathbb{C}), \\ P\left(H^{1}(X, \mathbb{C}) \times \mathfrak{g}\right) \cup \bigcup_{f \in \mathscr{E}_{X}} f^{!}\left(\mathscr{F}\left(H^{\bullet}(S, \mathbb{C}), \mathfrak{g}\right)\right) & \text { otherwise, }\end{cases}
$$

where, in the second case, $f: X \rightarrow S$ is an admissible map to a curve $S$ with $\chi(S)<0$, and likewise in the third case.

Proof. In all three cases, the stated equalities follow from Corollary 7.2, formula (55). The irreducibility of $P\left(H^{1}(X, \mathbb{C}) \times \mathfrak{g}\right)$ is clear, while the irreducibility of $f^{!}\left(\mathscr{F}\left(H^{\bullet}(S, \mathbb{C}), \mathfrak{g}\right)\right)$ follows from Lemma 7.3. It remains to check that there are no redundancies in the last case.

Let $f: X \rightarrow S$ and $f^{\prime}: X \rightarrow S^{\prime}$ be two admissible maps. In formula $(54)$, set $C=f^{*}\left(H^{1}(S, \mathbb{C})\right)$ and $C^{\prime}=f^{* *}\left(H^{1}\left(S^{\prime}, \mathbb{C}\right)\right)$, and assume $C \nsubseteq C^{\prime}$. Since $f^{!}\left(\mathscr{F}\left(H^{\bullet}(S, \mathbb{C}), \mathfrak{g}\right)\right)=\mathscr{F}\left(H^{\bullet}(X, \mathbb{C}), \mathfrak{g}\right) \cap$ $C \otimes \mathfrak{g}$, and similarly for $f^{\prime}$, we infer that $f^{!}\left(\mathscr{F}\left(H^{\bullet}(S, \mathbb{C}), \mathfrak{g}\right)\right) \nsubseteq f^{\prime !}\left(\mathscr{F}\left(H^{\bullet}\left(S^{\prime}, \mathbb{C}\right), \mathfrak{g}\right)\right)$.

Now, since $\mathscr{R}_{1}^{1}(X) \neq H^{1}(X, \mathbb{C})$, we must have $C \neq H^{1}(X, \mathbb{C})$. By a similar support argument, $P\left(H^{1}(X, \mathbb{C}) \times \mathfrak{g}\right) \nsubseteq f^{!}\left(\mathscr{F}\left(H^{\bullet}(S, \mathbb{C}), \mathfrak{g}\right)\right)$. Finally, recall from Lemma 7.3 that $f^{!}\left(\mathscr{F}\left(H^{\bullet}(S, \mathbb{C}), \mathfrak{g}\right)\right)$ contains regular elements. Hence, $f^{!}\left(\mathscr{F}\left(H^{\bullet}(S, \mathbb{C}), \mathfrak{g}\right)\right) \nsubseteq P\left(H^{1}(X, \mathbb{C}) \times \mathfrak{g}\right)$, and this completes the proof.

Theorem 7.5. Let $X$ be a 1-formal, quasi-projective manifold with $b_{1}(X)>0$, and let $\theta: \mathfrak{s l}_{2} \rightarrow$ $\mathfrak{g l}(V)$ be a finite-dimensional representation. Then, the decomposition into irreducible components of $\mathscr{R}_{1}^{1}\left(H^{\bullet}(X, \mathbb{C}), \theta\right)$ is given by

$$
\mathscr{R}_{1}^{1}\left(H^{\bullet}(X, \mathbb{C}), \theta\right)= \begin{cases}\Pi\left(H^{\bullet}(X, \mathbb{C}), \theta\right) & \text { if } \mathscr{R}_{1}^{1}(X)=\{0\}, \\ f^{!}\left(\mathscr{F}\left(H^{\bullet}(S, \mathbb{C}), \mathfrak{s l}_{2}\right)\right) & \text { if } \mathscr{R}_{1}^{1}(X)=H^{1}(X, \mathbb{C}), \\ \Pi\left(H^{\bullet}(X, \mathbb{C}), \theta\right) \cup \bigcup_{f \in \mathscr{E}_{X}} f^{!}\left(\mathscr{F}\left(H^{\bullet}(S, \mathbb{C}), \mathfrak{s l}_{2}\right)\right) & \text { otherwise, }\end{cases}
$$

where, in the second case, $f: X \rightarrow S$ is an admissible map to a curve $S$ with $\chi(S)<0$, and likewise in the third case.

Proof. In all three cases, the stated equalities follow from Corollary 7.2, formula (56). Furthermore, the irreducibility of $\Pi\left(H^{\bullet}(X, \mathbb{C}), \theta\right)$ follows from Lemma 3.9, while the irreducibility of $f^{!}\left(\mathscr{F}\left(H \bullet(S, \mathbb{C}), \mathfrak{s l}_{2}\right)\right)$ follows from Lemma 7.3 .

It remains to check that there are no redundancies in the last equality. In view of Theorem 7.4 , it is enough to verify that $\Pi\left(H^{\bullet}(X, \mathbb{C}), \theta\right) \nsubseteq f^{!}\left(\mathscr{F}\left(H^{\bullet}(S, \mathbb{C}), \mathfrak{s l}_{2}\right)\right)$, where $\theta$ is the inclusion $\mathfrak{s l}_{2} \hookrightarrow \mathfrak{g l}_{2}$. 
By our assumption on $\mathscr{R}_{1}^{1}(X)$, there is an element $\eta \in H^{1}(X, \mathbb{C}) \backslash f^{*}\left(H^{1}(S, \mathbb{C})\right)$. Pick a nonzero matrix $g \in \mathfrak{s l}_{2}$ such that $\operatorname{det}(\theta(g))=0$, and set $\omega=\eta \otimes g \in \Pi\left(H^{\bullet}(X, \mathbb{C}), \theta\right)$. Clearly, $\omega \notin f^{!}\left(\mathscr{F}\left(H^{\bullet}(S, \mathbb{C}), \mathfrak{s l}_{2}\right)\right)$, and so we are done.

7.6. Discussion. Let us compare the rank 1 irreducible decomposition (54) with the decomposition from Theorem 7.5. To that end, let $A=\left(H^{\bullet}(X, \mathbb{C}), d=0\right)$ be the formal model of a 1-formal, quasi-projective manifold with $b_{1}(X)>0$, and let $V_{\theta}$ be a finite-dimensional $\mathfrak{s l}_{2}$-module. Assuming $\mathscr{R}_{1}^{1}(X)$ is neither $\{0\}$, nor $A^{1}$, we see that the irreducible components of $\mathscr{R}_{1}^{1}(A, \theta)$ either arise by pullback along admissible maps, or are equal to $\Pi(A, \theta)$. When $\theta=\mathrm{id}_{\mathbb{C}}$, only the first possibility occurs.

Both in the rank 1 case and the nonabelian situation, geometric irreducible components (to be denoted $\mathrm{W}_{f}$ ) have the following property: There is an epimorphism of Lie algebras, $q: \mathfrak{h}(A) \rightarrow \mathfrak{k}$, such that $W_{f}=q^{*}\left(\operatorname{Hom}_{\text {Lie }}\left(\mathfrak{k}, \mathfrak{s l}_{2}\right)\right)$. This assertion may be verified as follows. First, note that $\mathrm{W}_{f}=f^{!}\left(\mathscr{F}\left(A(S), \mathfrak{s l}_{2}\right)\right)$, by construction, where $A(S)=\left(H^{\bullet}(S, \mathbb{C}), d=0\right)$ and $f^{*}: A^{\bullet}(S) \hookrightarrow A^{\bullet}$ is injective. Finally, we may use the natural identification from Proposition 4.5, and put $\mathfrak{k}=\mathfrak{h}(A(S))$ and $q=\mathfrak{h}\left(f^{*}\right)$.

Next, we note that the irreducible component $W=\Pi(A, \theta)$ has a different qualitative behavior from this pullback point of view. Indeed, let $A$ be an arbitrary 1-finite cdga with $H^{1}(A) \neq 0$ and let $\theta: \mathfrak{s l}_{2} \rightarrow \mathfrak{g l}_{2}$ be the inclusion map. We claim that there is no epimorphism $q: \mathfrak{h}(A) \rightarrow \mathfrak{k}$ such that $W=q^{*}\left(\operatorname{Hom}_{\text {Lie }}\left(\mathfrak{k}, \mathfrak{s l}_{2}\right)\right)$.

Indeed, using the definition of the holonomy Lie algebra we may write $\mathfrak{k}=\mathbf{L}\left(A_{1}\right) / R$, for some ideal $R$. Let $\operatorname{in}(R) \subseteq A_{1}$ be the vector subspace generated by the degree 1 components of all elements $r \in R$. If $\operatorname{in}(R) \neq A_{1}$, pick a basis $\left\{x_{1}, \ldots, x_{n}\right\}$ of $A_{1}$ such that $\left\{x_{1}, \ldots, x_{m}\right\}$, for some $m<n$, form a basis of $\operatorname{in}(R)$. Next, take a matrix $g \in \mathfrak{s l}_{2}$ with $\operatorname{det}(\theta(g)) \neq 0$, and define a morphism $\rho: \mathfrak{k} \rightarrow \mathfrak{s l}_{2}$ by setting $\rho\left(x_{i}\right)=0$ for $i \leqslant m$ and $\rho\left(x_{i}\right)=g$, for $i>m$. Clearly, $q^{*}(\rho) \notin W$. If $\operatorname{in}(R)=A_{1}$, the equality $W=q^{*}\left(\operatorname{Hom}_{\text {Lie }}\left(\mathfrak{k}, \mathfrak{s l}_{2}\right)\right)$ would imply $W=0$, by Proposition 4.5, thereby contradicting the assumption that $H^{1}(A) \neq 0$.

7.7. Positive weights. We now come back to the general quasi-projective case, as described in Proposition 7.1. The global results of Corlette and Simpson from [5], together with the local Theorem 2.9, suggest that the analysis of the parameter space for flat, $\mathfrak{g}$-valued connections on the Gysin model $A^{\bullet}$ is much more complicated than in the 1 -formal case, even when $\mathfrak{g}=\mathfrak{s} \mathfrak{l}_{2}$.

A useful feature of Gysin models is the existence of a weight decomposition with weights 1 and 2 in degree 1 (cf. Morgan [16]). This means we have a direct sum decomposition, $A^{\bullet}=\bigoplus_{j \in \mathbb{Z}} A_{j}^{\bullet}$, such that $A_{j}^{\bullet} \cdot A_{k}^{\bullet} \subseteq A_{j+k}^{\bullet}, d A_{j}^{\bullet} \subseteq A_{j}^{\bullet}$ and $A_{j}^{1}=0$ for $j \neq 1,2$. Our next result implies that inclusion (52) becomes an equality for $\mathfrak{g}=\mathfrak{s l}_{2}$ or $\mathfrak{s o l}_{2}$, in the case when $X$ is quasi-projective and $\mathscr{R}_{1}^{1}(X)=\{0\}$. Indeed, Theorem 2.10 guarantees that $\mathscr{R}_{1}^{1}(A)=\{0\}$, hence the right hand side in (52) reduces to $\mathscr{F}^{1}(A, \mathfrak{g})$.

Proposition 7.6. Let $A^{\bullet}$ be a connected cdga admitting a weight decomposition with weights 1 and 2 in degree 1 , and suppose $\mathscr{R}_{1}^{1}\left(H^{\bullet}(A)\right) \subseteq\{0\}$. Then $\mathscr{F}(A, \mathfrak{g})=\mathscr{F}^{1}(A, \mathfrak{g})$, for $\mathfrak{g}=\mathfrak{s l}_{2}$ or $\mathfrak{s o l}_{2}$.

Proof. We first treat the case $\mathfrak{g}=\mathfrak{s l}_{2}$. An arbitrary element $\omega \in \mathscr{F}(A, \mathfrak{g})$ can then be written as $\alpha \otimes X+\beta \otimes Y+\gamma \otimes H$, with $\alpha, \beta, \gamma \in A^{1}$. It is readily checked that the Maurer-Cartan equation (10) is now equivalent to the following system of equations:

$$
d \gamma+\alpha \beta=0, d \alpha+2 \gamma \alpha=0, d \beta-2 \gamma \beta=0
$$


We have to show that $\operatorname{rank}\{\alpha, \beta, \gamma\} \leqslant 1$. To this end, let $\alpha=\alpha_{1}+\alpha_{2}, \beta=\beta_{1}+\beta_{2}$, and $\gamma=\gamma_{1}+\gamma_{2}$ be the respective weight decompositions. The weight 1 part of (58) is

$$
d \alpha_{1}=d \beta_{1}=d \gamma_{1}=0
$$

The weight 2 component is

$$
d \gamma_{2}+\alpha_{1} \beta_{1}=d \alpha_{2}+2 \gamma_{1} \alpha_{1}=d \beta_{2}-2 \gamma_{1} \beta_{1}=0
$$

For the weight 3 component we find that $\alpha_{1} \beta_{2}-\beta_{1} \alpha_{2}=\gamma_{1} \alpha_{2}-\alpha_{1} \gamma_{2}=\gamma_{1} \beta_{2}-\beta_{1} \gamma_{2}=0$, and, finally, the weight 4 component gives

$$
\alpha_{2} \beta_{2}=\gamma_{2} \alpha_{2}=\gamma_{2} \beta_{2}=0 .
$$

Using our hypothesis on $\mathscr{R}_{1}^{1}\left(H^{\bullet}(A)\right)$, we infer from (59) and (60) that $\operatorname{rank}\left\{\alpha_{1}, \beta_{1}, \gamma_{1}\right\} \leqslant 1$ and $d \alpha_{2}=d \beta_{2}=d \gamma_{2}=0$. Using (61), the same argument implies that $\operatorname{rank}\left\{\alpha_{2}, \beta_{2}, \gamma_{2}\right\} \leqslant 1$. If $\operatorname{rank}\left\{\alpha_{1}, \beta_{1}, \gamma_{1}\right\}=0$ (respectively, $\operatorname{rank}\left\{\alpha_{2}, \beta_{2}, \gamma_{2}\right\}=0$ ), we are done. Thus, we may assume that $\alpha_{1}=a \omega_{1}, \beta_{1}=b \omega_{1}, \gamma_{1}=c \omega_{1}$, with $0 \neq \omega_{1} \in A_{1}^{1}, d \omega_{1}=0$ and $(a, b, c) \neq 0$. Similarly, we may write $\alpha_{2}=a^{\prime} \omega_{2}, \beta_{2}=b^{\prime} \omega_{2}, \gamma_{2}=c^{\prime} \omega_{2}$, with $0 \neq \omega_{2} \in A_{2}^{1}$, d $\omega_{2}=0$, and $\left(a^{\prime}, b^{\prime}, c^{\prime}\right) \neq 0$.

Using (58), we deduce that either $\omega_{1} \omega_{2}=0$, or $a b^{\prime}-b a^{\prime}=c a^{\prime}-a c^{\prime}=c b^{\prime}-b c^{\prime}=0$. In the first case, we must have $\operatorname{rank}\left\{\omega_{1}, \omega_{2}\right\}=2$, contradicting the assumption that $\mathscr{R}_{1}^{1}\left(H^{\bullet}(A)\right) \subseteq\{0\}$. In the second case, $\left(a^{\prime}, b^{\prime}, c^{\prime}\right)=\lambda(a, b, c)$, which implies $\omega \in \mathscr{F}^{1}(A, \mathfrak{g})$ and completes the proof in the case when $\mathfrak{g}=\mathfrak{s l}_{2}$.

When $\mathfrak{g}=\mathfrak{s o l}_{2}$, an element $\omega \in \mathscr{F}(A, \mathfrak{g})$ may be written as $\omega=\alpha \otimes h+\beta \otimes x$, with $\alpha, \beta \in A^{1}$. In this case, the Maurer-Cartan equation (10) is equivalent to the system of equations $d \alpha=0$ and $d \beta+\alpha \beta=0$. An argument as before shows that $\operatorname{rank}\{\alpha, \beta\} \leqslant 1$, thereby completing the proof.

We obtain as a corollary another class of examples where higher rank embedded jump loci are controlled by information on the corresponding rank 1 loci.

Corollary 7.7. Let $X$ be a quasi-projective manifold with $b_{1}(X)>0$, and let $\theta: \mathfrak{g} \rightarrow \mathfrak{g l}(V)$ be a finite-dimensional representation, where $\mathfrak{g}=\mathfrak{s l}_{2}$ or $\mathfrak{s o l}_{2}$. Assume $\mathscr{R}_{1}^{1}(X)=\{0\}$. Then the inclusions from Proposition 7.1 become equalities, for any Gysin model A of X. More precisely,

$$
\mathscr{F}(A, \mathfrak{g})=\mathscr{F}^{1}(A, \mathfrak{g}) \quad \text { and } \quad \mathscr{R}_{1}^{1}(A, \theta)=\Pi(A, \theta) .
$$

Proof. By Theorem 2.10, $\mathscr{R}_{1}^{1}(A)=\{0\}$; thus, by formula $(50), \mathscr{E}_{X}=\varnothing$. Hence, all we have to do is to prove equalities (62).

The first equality follows from Proposition 7.6, while the inclusion $\Pi(A, \theta) \subseteq \mathscr{R}_{1}^{1}(A, \theta)$ follows from Corollary 3.8. To check the reverse inclusion, pick $0 \neq \omega=\eta \otimes g \in \mathscr{R}_{1}^{1}(A, \theta)$. Again by Corollary 3.8, we find an eigenvalue $\lambda$ of $\theta(g)$ such that $\lambda \eta \in \mathscr{R}_{1}^{1}(A)$. Hence, $\lambda=0$, and therefore $\omega \in \Pi(A, \theta)$.

For a detailed study of algebras having vanishing resonance, we refer to [19]. Finally, it should be noted that the classical, depth 1 resonance varieties behave well under finite products and coproducts, see $[18, \S 13]$. Using some of the machinery developed in this paper, we show in [21] that analogous product and coproduct formulas hold in the non-abelian setting.

Acknowledgments. Some of this work was done while S. Papadima and A. Suciu visited the Max Planck Institute for Mathematics in Bonn in April-May 2012. The work was continued while A.S. visited the Institute of Mathematics of the Romanian Academy in June, 2012 and June, 2013. The work was completed while A.S. visited again MPIM Bonn in September-October 
2013. Thanks are due to both institutions for their hospitality, support, and excellent research atmosphere. We also thank the two referees for their useful remarks and suggestions.

\section{REFERENCES}

[1] D. Arapura, Geometry of cohomology support loci for local systems I, J. Algebraic Geom. 6 (1997), no. 3, 563-597. MR1487227 1.2, 7.1

[2] R. Body, M. Mimura, H. Shiga, D. Sullivan, p-universal spaces and rational homotopy types, Comment. Math. Helv. 73 (1998), 427-442. MR1633367 2.6

[3] K.-T. Chen, Extension of $C^{\infty}$ function algebra by integrals and Malcev completion of $\pi_{1}$, Advances in Math. 23 (1977), no. 2, 181-210. MR045846 4.3

[4] C. Chevalley, S. Eilenberg, Cohomology theory of Lie groups and Lie algebras, Trans. Amer. Math. Soc. 63 (1948), 85-124. MR0024908 2.13

[5] K. Corlette, C. Simpson, On the classification of rank-two representations of quasiprojective fundamental groups, Compos. Math. 144 (2008), no. 5, 1271-1331. MR2457528 1.1, 7.7

[6] A. Dimca, S. Papadima, Nonabelian cohomology jump loci from an analytic viewpoint, Commun. Contemp. Math. 16 (2014), no. 4, 1350025 (47 pp). MR3231055 1.2, 1.3, 1.4, 1.7, 1.9, 2.1, 2.8, 2.6, 2.9, 2.6, 2.6, 2.7, $2.13,4.4,4.6,5,7.1$

[7] A. Dimca, S. Papadima, A. I. Suciu, Topology and geometry of cohomology jump loci, Duke Math. Journal 148 (2009), no. 3, 405-457. MR2527322 1.2, 1.3, 1.6, 1.7, 1.8, 1.9, 2.6, 4, 4.2, 4.4, 4.4, 5, 5, 6.6, 6.6, 7.3

[8] J. Dixmier, Cohomologie des algèbres de Lie nilpotentes, Acta Sci. Math. Szeged 16 (1955), 246-250. MR0074780 4.7, 4.16

[9] W. Fulton, J. Harris, Representation theory, Grad. Texts in Math., vol. 129, Springer-Verlag, New York, 1991. MR1153249 3.3

[10] W. Goldman, J. Millson, The deformation theory of representations of fundamental groups of compact Kähler manifolds, Inst. Hautes Études Sci. Publ. Math. 67 (1988), 43-96. MR0972343 1.1, 2.2

[11] P. J. Hilton, U. Stammbach, A course in homological algebra, Grad Texts in Math., vol. 4, Springer-Verlag, New York, 1971. MR0346025 4.3

[12] J. E. Humphreys, Introduction to Lie algebras and representation theory, Grad. Texts in Math., vol. 9, Springer-Verlag, New York, 1972. MR0323842 3.3, 6.5

[13] M. Kapovich, J. Millson, On representation varieties of Artin groups, projective arrangements and the fundamental groups of smooth complex algebraic varieties, Inst. Hautes Études Sci. Publ. Math. 88 (1998), 5-95. MR1733326 1.1, 1.8, 1.8, 6.1, 6.7, 6.7, 6.7

[14] A. Libgober, First order deformations for rank one local systems with a non-vanishing cohomology, Topology Appl. 118 (2002), no. 1-2, 159-168. MR1877722 1.4, 2.6

[15] J. Milnor, D. Husemoller, Symmetric bilinear forms, Ergebnisse der Mathematik und ihrer Grenzgebiete, Band 73, Springer-Verlag, New York-Heidelberg, 1973. MR0506372 7.4

[16] J. W. Morgan, The algebraic topology of smooth algebraic varieties, Inst. Hautes Études Sci. Publ. Math. 48 (1978), 137-204. MR0516917 1.4, 2.12, 7.7

[17] S. Papadima, A. Suciu, Algebraic invariants for right-angled Artin groups, Math. Annalen 334 (2006), no. 3, 533-555. MR2207874 1.8, 6.1

[18] S. Papadima, A. Suciu, Bieri-Neumann-Strebel-Renz invariants and homology jumping loci, Proc. London Math. Soc. 100 (2010), no. 3, 795-834. MR2640291 1.2, 2.1, 7.7

[19] S. Papadima, A. Suciu, Vanishing resonance and representations of Lie algebras, to appear in Journal für die reine und angewandte Mathematik (Crelle's Journal) at doi:10.1515/crelle-2013-0073, available at arxiv: $1207.2038 \mathrm{v} 2.7 .7$

[20] S. Papadima, A. Suciu, Jump loci in the equivariant spectral sequence, Math. Res. Lett. 21 (2014), no. 4, 863-883. MR3275650 2.1, 2.6

[21] S. Papadima, A. Suciu, Non-abelian resonance: product and coproduct formulas, in Experimental and Theoretical Methods in Algebra, Geometry and Topology, Springer Proceedings in Mathematics and Statistics, vol. 96, 2014, pp. 269-280. 7.7

[22] S. Papadima, A. Suciu, The Milnor fibration of a hyperplane arrangement: from modular resonance to algebraic monodromy, preprint arxiv:1401.0868v2. 1.9

[23] D. Quillen, Rational homotopy theory, Ann. of Math. 90 (1969), 205-295. MR0258031 2.11, 2.13 
[24] A. Suciu, Characteristic varieties and Betti numbers of free abelian covers, Intern. Math. Res. Notices 2014 (2014), no. 4, 1063-1124. MR3168402 1.2

[25] D. Sullivan, Infinitesimal computations in topology, Inst. Hautes Études Sci. Publ. Math. 47 (1977), $269-331$. MR0646078 2.6

Simion Stoilow Institute of Mathematics, P.O. Box 1-764, RO-014700 Bucharest, Romania E-mail address: Anca.Macinic@imar.ro

Simion Stollow Institute of Mathematics, P.O. Box 1-764, RO-014700 Bucharest, Romania E-mail address: Stefan.Papadima@imar.ro

Simion Stollow Institute of Mathematics, P.O. Box 1-764, RO-014700 Bucharest, Romania

E-mail address: Radu.Popescu@imar.ro

Department of Mathematics, Northeastern University, Boston, MA 02115, USA

E-mail address: a.suciu@neu.edu 